\title{
Scaling laws for mixing and dissipation in unforced rotating stratified turbulence
}

\author{
A. Pouquet ${ }^{1,2}$, D. Rosenberg ${ }^{3}$, R. Marino ${ }^{4}$ and C. Herbert ${ }^{5}$ \\ ${ }^{1}$ National Center for Atmospheric Research, P.O. Box 3000, Boulder, CO, 80307, USA. \\ ${ }^{2}$ Atmospheric \& Space Physics Laboratory, University of Colorado, Boulder CO, 80309, USA. \\ ${ }^{3}$ duaner62@gmail.com. \\ ${ }^{4}$ Laboratoire de Mécanique des Fluides et d'Acoustique, CNRS, École Centrale de Lyon, \\ Université de Lyon, Écully, 69134, FRANCE. \\ ${ }^{5}$ École Normale Supérieure, 46 Allée d'Italie, Lyon, F-69364, FRANCE.
}

(Received $\mathrm{xx}$; revised $\mathrm{xx}$; accepted $\mathrm{xx}$ )

We present a model for the scaling of mixing in weakly rotating stratified flows characterized by their Rossby, Froude and Reynolds numbers $R o, F r, R e$. It is based on quasiequipartition between kinetic and potential modes, sub-dominant vertical velocity $w$, and lessening of the energy transfer to small scales as measured by a dissipation efficiency $\beta=\epsilon_{V} / \epsilon_{D}$, with $\epsilon_{V}$ the kinetic energy dissipation and $\epsilon_{D}=u_{r m s}^{3} / L_{\text {int }}$ its dimensional expression, $w, u_{r m s}$ the vertical and $r m s$ velocities, and $L_{i n t}$ the integral scale. We determine the domains of validity of such laws for a large numerical study of the unforced Boussinesq equations mostly on grids of $1024^{3}$ points, with $R o / F r \geqslant 2.5$, and with $1600 \leqslant R e \approx 5.4 \times 10^{4}$; the Prandtl number is one, initial conditions are either isotropic and at large scale for the velocity, and zero for the temperature $\theta$, or in geostrophic balance. Three regimes in Froude number, as for stratified flows, are observed: dominant waves, eddy-wave interactions and strong turbulence. A wave-turbulence balance for the transfer time $\tau_{t r}=N \tau_{N L}^{2}$, with $\tau_{N L}=L_{i n t} / u_{r m s}$ the turn-over time and $N$ the BruntVäisälä frequency, leads to $\beta$ growing linearly with $F r$ in the intermediate regime, with a saturation at $\beta \approx 0.3$ or more, depending on initial conditions for larger Froude numbers. The Ellison scale is also found to scale linearly with $F r$. The flux Richardson number $R_{f}=B_{f} /\left[B_{f}+\epsilon_{V}\right]$, with $B_{f}=N\langle w \theta\rangle$ the buoyancy flux, transitions for roughly the same parameter values as for $\beta$. These regimes for the present study are delimited by $\mathcal{R}_{B}=\operatorname{ReFr}^{2} \approx 2$ and $R_{B} \approx 200$. With $\Gamma_{f}=R_{f} /\left[1-R_{f}\right]$ the mixing efficiency, putting together the three relationships of the model allows for the prediction of the scaling $\Gamma_{f} \sim F^{-2} \sim \mathcal{R}_{B}^{-1}$ in the low and intermediate regimes for high $R e$, whereas for higher Froude numbers, $\Gamma_{f} \sim \mathcal{R}_{B}^{-1 / 2}$, a scaling already found in observations: as turbulence strengthens, $\beta \sim 1, w \approx u_{r m s}$, and smaller buoyancy fluxes altogether correspond to a decoupling of velocity and temperature fluctuations, the latter becoming passive.

\section{Introduction}

Mixing, which takes place for a large domain of flow parameters in fully developed turbulence (FDT), in engineering and geophysical flows, has been analyzed extensively (Peltier \& Caulfield 2003; Dimotakis 2005; Ivey et al. 2008). One central issue is that of mixing efficiency, which can be defined in many ways (see Mashayek et al. (2017) and references therein), for example as the ratio in the momentum equation of the buoyancy flux to the rate of kinetic energy dissipation (see $\$ 4$ for more details). For example, in the ocean, it affects chemistry and plankton dynamics, as well as the global circulation and hence the climate on long time-scales (Ivey et al. 2008; McWilliams 2016). The mean 
circulation of the ocean and atmosphere is modified by a combination of stratification and dissipation, but rotation can also play a role. In the oceanic context, data indicates an enhanced vertical mixing that can be compared with the Osborn (1980) model, stating that the adimensionalized scalar diffusivity is proportional to the turbulence intensity parameter, $\mathcal{R}_{I}=\epsilon_{V} /\left[\nu N^{2}\right]$, with an efficiency $\Gamma_{f}=B_{f} / \epsilon_{V} \approx 0.2$ as soon as $\mathcal{R}_{I} \gtrsim 10$ (see also Lindborg \& Brethouwer (2008); Karimpour \& Venayagamoorthy (2015)); $\epsilon_{V}$ is the energy dissipation rate, $\nu$ the kinematic viscosity, $B_{f}$ the buoyancy flux, and $N$ is the Brunt-Väisälä frequency. Unsurprisingly, though, there is evidence that this efficiency coefficient does depend on the intensity of the turbulence within a stratified flow (Smyth et al. 2001; Ivey et al. 2008), and exhibits temporal variability associated with secondary instabilities of Kelvin-Helmoltz rolls (Mashayek \& Peltier 2013), as well as a variation with Prandtl number (Smyth et al. 2001). Indeed, mixing efficiency is in part governed by secondary instabilities that develop three-dimensional eddies at high Reynolds numbers. These eddies can dominate the whole process irrespective of Froude number but in ways that may depend on other parameters of the flow, e.g., in the presence or not of shear, leading to a lack of monotonicity in its variation with the Richardson number (Peltier \& Caulfield 2003). Thus, a one-parameter modeling of such flows based on stratification alone may be insufficient.

The amount of kinetic energy available for dissipation at small scales in rotating stratified turbulence (RST) is a crucial quantity for sub-grid scale parameterizations in oceanic and climate models, and depends on the amount of energy that is transferred to these small scales in the presence of inertia-gravity waves, through breaking at small scales of the large-scale quasi-geostrophic (QG) balance (Lelong \& Riley 1991; Staquet \& Sommeria 2002; Riley \& deBruynKops 2003), and a lowering of the Richardson number below some critical value. Wave-turbulence interactions allow for coupling to the mean flow (Finnigan 1999); they have been measured in the stratosphere as well as in the upper ocean and lead to vertical mixing and enhanced dissipation (see e.g., Klymak et al. (2008); van Haren et al. (2016)). Few studies have considered mixing in decaying rotating stratified flows. Lagrangian diffusion in RST is studied in Cambon et al. (2004); with $N / f$ taking the values of $0.1,1$ and 10 , as well as 0 and $\infty$, a reduced diffusivity is found in the vertical, but not in the horizontal. The role of $N / f$, with $f$ twice the rotation rate, as a governing parameter for the intensity of lateral mixing with geostrophic adjustment has also been stressed in Lelong \& Sundermeyer (2005). Experimentally, it was shown in Praud et al. (2006) that structures develop an aspect ratio proportional to $f / N$ (see also Waite \& Bartello (2006); Kurien \& Smith (2014) for direct numerical simulations for the forced case): in RST, there is a progressive shift from a vertical scale due entirely to stratification, the buoyancy scale $L_{B}=u_{r m s} / N$, to one corresponding to QG where rotation and stratification are in balance with pressure gradients. Finally, Dritschel \& McKiver (2015) studied the influence of $N / f$ on large-scale quasi-geostrophic balance; they found it weak, the flows remaining balanced throughout the studied parameter regime, although vertical velocity increases with $f / N$ for $F r^{2}<<R o<<1$.

It is in this context that we now analyze several sets of direct numerical simulations (DNS) of decaying RST, motivated by atmospheric and oceanic applications, with Reynolds numbers up to $R e \approx 1.85 \times 10^{4}$, an upper value comparable to that in the Mesosphere and Lower Thermosphere (Liu et al. 2013). We show that three simple scaling laws for the potential to kinetic energy ratio, the vertical to kinetic energy ratio, and the effective dissipation coefficient together lead to the recovery of a well-known scaling for mixing as a function of Froude number at high Reynolds numbers, and that these scalings persist even when significantly different initial conditions-i.e., those in quasi-geostrophic balance- are used. 


\section{Problem setting}

The Boussinesq equations, with constant rotation and stable stratification are:

$$
\begin{aligned}
\partial_{t} \mathbf{u}+\nabla p+\mathbf{u} \cdot \nabla \mathbf{u} & =f \mathbf{u} \times \hat{z}-N \theta \hat{z}+\nu \delta \mathbf{u}, \\
\partial_{t} \theta+\mathbf{u} \cdot \nabla \theta & =N w+\kappa \delta \theta,
\end{aligned}
$$

with $\mathbf{u}=(u, v, w)$ the incompressible velocity field, $\nabla \cdot \mathbf{u}=0$, and $p$ the pressure; $\theta$ represents temperature (or density) fluctuations, in units of a velocity, since we want to stress the energetics of these flows. These fluctuations are super-imposed on on a stably stratified background with a linear vertical profile $\bar{\theta}(z)=\theta_{0}+z \partial_{z} \bar{\theta}, \partial_{z} \bar{\theta}<0$. Introducing the buoyancy $b=N \theta$ (note the choice of sign), with point-wise vertical flux $b(\mathbf{x}) w(\mathbf{x})$, as well as the integrated buoyancy flux

$$
B_{f}=\langle N \theta w\rangle,
$$

one recovers the more standard form of the equations in terms of $b . N=\sqrt{-\left(g / \theta_{0}\right) \partial_{z} \bar{\theta}}$ is the Brunt-Väisälä frequency, and $\nu=\kappa$ are the viscosity and thermal diffusivity. The Boussinesq equations are integrated using direct numerical simulations. A cubic box of $n_{p}^{3}=1024^{3}$ points is used for 56 runs (see Table 11, with a linear dimension $L_{b o x}=2 \pi$, resulting in wave numbers in the range $1 \leqslant k \leqslant k_{M}=n_{p} / 3$ using a standard $2 / 3$ dealiasing rule. All length scales defined below are thus expressed in terms of the fundamental length $L_{b o x}=2 \pi$, which of course can be rescaled to the physical problem when necessary. Another smaller set of runs, at lower resolutions, is also analyzed (see Table 2). The pseudo-spectral code we use, GHOST (Geophysical High-Order Suite for Turbulence), is parallelized in a hybrid fashion with both MPI and Open-MP (Mininni et al. 2011) and demonstrates scalability to in excess of 130,000 cores. It includes many solvers for fluid and magnetohydrodynamic turbulence, and it now also has the capability to simulate non-cubic geometry (Mininni \& Pouquet 2017). GHOST has been tested in the purely stratified case against the numerical results of Kimura \& Herring (1996) for random initial conditions, and of Riley \& deBruynKops (2003) for the Taylor-Green flow.

For the runs of Table 1, the potential energy is initially zero, and initial conditions for the initial velocity, with a non-zero vertical component, are isotropic, random and centered on the large scales $\left(2 \pi / L_{0}=k_{0}=2.5\right)$; energies are defined as:

$$
E_{V}=\frac{1}{2} \int\|\mathbf{u}(\mathbf{x})\|^{2} d^{3} \mathbf{x}, \quad E_{P}=\frac{1}{2} \int|\theta(\mathbf{x})|^{2} d^{3} \mathbf{x}, \quad E_{T}=E_{V}+E_{P},
$$

$E_{T}$ being the total energy. They can also be written in terms of their respective isotropic Fourier spectra, with $\int E_{V, P}(k) d k=E_{V, P}$. Similarly, the kinetic, potential and total rates of energy dissipation are:

$$
\epsilon_{V}=\nu \int\|\boldsymbol{\omega}(\mathbf{x})\|^{2} d^{3} \mathbf{x}, \quad \epsilon_{P}=\kappa \int\|\nabla \theta(\mathbf{x})\|^{2} d^{3} \mathbf{x}, \quad \epsilon_{T}=\epsilon_{V}+\epsilon_{P} .
$$

Note that $\epsilon_{V}$ can be measured relative to its dimensional evaluation of kinetic energy dissipation for a fully turbulent flow as:

$$
\beta \equiv \epsilon_{V} / \epsilon_{D}, \epsilon_{D} \equiv u_{r m s}^{3} / L_{i n t}, u_{r m s}=\left[\left\langle|\mathbf{u}|^{2}\right\rangle\right]^{1 / 2}, L_{i n t}=2 \pi \frac{\int\left[E_{V}(k) / k\right] d k}{\int E_{V}(k) d k},
$$

where $L_{\text {int }}$ is the integral scale (Monin \& Yaglom 1979). $\beta$ is a key parameter of the phenomenological and theoretical understanding of the interactions between waves and turbulence (Zakharov et al. 1992), as also discussed in $\$ 3.3$

The governing dimensionless parameters of the Boussinesq equations measure the 
strength of nonlinear interactions relative to dissipation, rotation and stratification; they are the Reynolds (Re), Rossby (Ro) and Froude (Fr) numbers, defined as usual as:

$$
R e=\frac{u_{r m s} L_{i n t}}{\nu}, R o=\frac{u_{r m s}}{f L_{i n t}}, F r=\frac{u_{r m s}}{N L_{i n t}},
$$

with the Prandtl number $\operatorname{Pr}=\nu / \kappa$ taken equal to unity. The buoyancy Reynolds number, the Richardson number, and the turbulent intensity are defined as:

$$
\mathcal{R}_{B} \equiv R e F r^{2}, \quad R i \equiv\left[N /\left\langle\partial_{z} u_{\perp}\right\rangle\right]^{2}, \quad \mathcal{R}_{I} \equiv \epsilon_{V} /\left[\nu N^{2}\right]
$$

$R i$ is based on a shear time computed on vertical gradients of the horizontal wind, namely $\tau_{\text {shear }}=\left[\left\langle\partial_{z} u_{\perp}\right\rangle\right]^{-1}$. All these parameters are discussed further in the Appendix, $\$ 9.2$ In the presence of stratification, a variety of length-scales can be relevant (Thorpe 1987; Mater \& Venayagamoorthy 2014), e.g.

$$
L_{B}=2 \pi \sqrt{E_{V}} / N, L_{E l l}=2 \pi \sqrt{E_{P}} / N, \ell_{O z}=2 \pi \sqrt{\epsilon_{V} / N^{3}},
$$

or the buoyancy, Ellison and Ozmidov scales. In purely stratified flows, $L_{B}$ is the scale for which the vertical Froude number becomes of order one (Billant \& Chomaz 2001); it measures the thickness of the vertical layers. On the other hand, the Ellison scale corresponds to the vertical distance traveled by a particle of fluid before being completely mixed, and it is thought to be significantly smaller than the integral scale in strongly stratified flows, as we shall show later (see Figs. 6p). $L_{B}$ and $L_{E l l}$ vary as $1 / N$, but differ by a $\sqrt{E_{V} / E_{P}}$ field-amplitude ratio. Finally, the Ozmidov scale is the scale beyond which isotropy is thought to be recovered together with a classical Kolmogorov range.

\section{Global behavior and scaling}

\subsection{Overview of the runs}

Runs with an emphasis on realistic parameters for the mesosphere and lower thermosphere, and that overlap with the present data base, were investigated for the energy partition between waves and slow modes and the link with kinetic-potential energy exchanges in Marino et al. (2015b), as well as for parametric characteristic time-scale variations in Rosenberg et al. (2016) (see also Rosenberg et al. (2017)). Here, the runs on grids of $1024^{3}$ points, cover the following parameter ranges (see Table 1 ): $0.11 \leqslant R o \leqslant 41$, $1985 \leqslant R e \leqslant 18590,0.001 \leqslant F r \leqslant 5.5,0.02<\mathcal{R}_{B}<1.2 \times 10^{5}$ and $2.47 \leqslant N / f \leqslant 312$. Two purely stratified runs are included as well. Note that, even though the ratio in values of Reynolds numbers across all these runs is close to ten, most runs are within a factor $\approx 4$ of each other in $R e$, with as high a value as can be realized on the chosen grid, thus breaking large-scale balance toward isotropization, as studied already in Herring (1980) using a closure model of turbulence (see Pumir et al. (2016); Rubinstein et al. (2017) and Iyer et al. (2017) for recent references).

Two other small series of runs at lower resolutions have been performed (see Table 2). The first study ( $\mathbf{Q}$ runs) is focused on the role of initial conditions, taking now geostrophically balanced fields at $t=0$, which should radiate waves much less initially. The second small set of $(\mathbf{Z})$ runs deals with the variation of effective dissipation $\beta$ defined in equation 2.5 with Reynolds number at fixed $N / f=5$, with $R e$ varying by a factor in excess of 10, between 1650 and 18590, when including runs of Table 1 .

All statistics are computed dynamically around the peak of dissipation, when the flow is most developed and starts its self-similar temporal decay. This is in contrast to what is done in Stretch et al. (2010), where the data for mixing is taken when more than 
$90 \%$ of the energy has dissipated, after roughly ten turnover times. Specifically, our data is averaged on a number of outputs around the peak of enstrophy, covering a relative variation in the amplitude of enstrophy of $\approx 2.5 \%$. This results in using of the order of 18 outputs on average for each run, with no more than 49 and no fewer than 6 ; the physical time interval on which these averages were performed is of the order of a fraction of a turn-over time. We find that $u_{r m s}$ and $L_{\text {int }}$ do not vary much across the first large parametric study, from 0.66 to 0.89 for the former, and from 1.39 to 2.78 for the latter. Note that some of the runs tabulated in Rosenberg et al. (2016) have been removed from the data set in Table 1. which has been reduced from 65 to 56 runs. This is because of various factors: archiving issues in view of the large data base that was created several years ago, or because the variation of enstrophy at peak was insufficient to satisfy the averaging criterion, or because some of the data files were corrupted.

The accuracy of the computations is quantified through the ratio of the maximum to the Kolmogorov dissipation wavenumber, $k_{M} / k_{\eta}$, with $k_{\eta}=\left[\epsilon_{V} / \nu^{3}\right]^{1 / 4}$; this is done under the assumption that the small scales have recovered a Kolmogorov spectrum, i.e. that the Ozmidov length scale is resolved, or for $\mathcal{R}_{B} \geqslant 1$, which is the case for the majority of our runs. For all flows of Table 1 . we have $0.39 \leqslant k_{\eta} / k_{M} \leqslant 1.3$, with roughly $17 \%$ slightly under-resolved runs which all have $N / f \geqslant 10$. We have also checked that the overall shape of the curves plotted in the figures did not depend on the resolution.

Strong activity develops at small scales, with layer destabilization, as found as well by a number of authors in the purely stratified case. An example of such structures is given in the visualizations found in Rosenberg et al. (2015) for a flow which corresponds rather closely to some of the runs computed in this data base (specifically, run $\mathrm{Id}=11$, 19, 33 and 43), but done on a grid of $4096^{3}$ points, allowing for a substantially higher Reynolds number. Prominent in this flow with $N / f=4.95, F r \approx 0.0242, R e \approx 54000$, is the juxtaposition of large-scale eddies and an intense vortical activity at their rims in what can be called vortex lanes. Such a complex small-scale flow corresponds to local overturning instabilities, with local Richardson numbers well below 0.25 for a substantial portion of the flow (see e.g. Figs. 10 and 11 in Rosenberg et al. (2015)).

In all the figures in the present work, different symbols are used for different binning, mainly in Rossby number; stars/asterisks are used for runs with QG initial conditions (ICs), whereas the hollow shapes are always for the $\theta(t=0)=0$ ICs. Furthermore, the sizes of all symbols refer to the resolution and Reynolds number (see caption of Fig. 1).

\subsection{Energy ratios}

We show in Fig. 1 the variation of central energetic quantities, as a function of Froude number, with binning in Rossby number. The widths of the bins are chosen so as to have approximately the same number of data points in each bin, as for all other figures. The energy ratio $r_{E} \equiv E_{P} / E_{V}$ (left) varies roughly between 0.2 and 0.4 , as long as the velocity and temperature remain coupled through buoyancy, i.e. for $F r<1$. At high $F r, E_{P} / E_{V}$ becomes negligible since the velocity is no longer constrained effectively by the waves and we have a quasi passive scalar regime with $\theta(t=0)=0$ for most of the runs. The scaling in $\mathrm{Fr}^{-2}$ at high $\mathrm{Fr}$, as advocated for oceanic turbulence in Wells et al. (2010) (see also Maffioli et al. (2016) for purely stratified flows), may be present as well, although we have a scarcity of points in that domain. We thus conclude that in the intermediate regime of wave-vortex interactions:

$$
\theta_{r m s} \sim u_{r m s} .
$$

The result $r_{E} \approx 1$ from below (see also Mater et al. $(2013)$ ) is compatible with the experimental, observational and numerical data compiled in Zilitinkevich et al. (2008). It 
TABLE 1. Nomenclature of the runs performed on grids of $1024^{3}$ points, with Id, Fr, Ro and $R e$ the identification of runs and their Froude, Rossby and Reynolds numbers, the runs being ordered by $\mathrm{Fr}$ (see also Rosenberg et al. (2016)). Initial conditions are centered on the large scales and are isotropic for the velocity field, and zero for the temperature. Runs 62 to 65 are purely stratified. Note that the 9 runs marked with a star are not included in the present study (see 3.1 ; ; they are specifically runs $\mathrm{Id}=4,8,10,21,30,38,50,62$ and 63 .

\begin{tabular}{|c|c|c|c|c|c|c|c|c|c|c|c|c|c|}
\hline Id & $F r$ & Ro & $R e$ & - & Id & $F r$ & Ro & $R e$ & - & Id & $F r$ & Ro & $R e$ \\
\hline 1 & 0.0013 & 0.129 & 10905 & & 2 & 0.0023 & 0.115 & 9895 & & 3 & 0.0061 & 0.120 & 10680 \\
\hline $4^{*}$ & 0.0064 & 0.633 & 9270 & . & 5 & 0.0073 & 0.225 & 13945 & . & 6 & 0.0116 & 0.305 & 14680 \\
\hline 7 & 0.0119 & 2.98 & 13500 & . & $8^{*}$ & 0.0127 & 0.635 & 8930 & . & 9 & 0.021 & 0.147 & 11080 \\
\hline $10 *$ & 0.0215 & 0.464 & 13450 & . & 11 & 0.022 & 0.116 & 10980 & . & 12 & 0.0262 & 4.58 & 10980 \\
\hline 13 & 0.028 & 0.14 & 10720 & . & 14 & 0.030 & 9.4 & 10520 & . & 15 & 0.033 & 4.58 & 13020 \\
\hline 16 & 0.036 & 9.1 & 13200 & . & 17 & 0.038 & 0.140 & 10530 & . & 18 & 0.041 & 0.607 & 9840 \\
\hline 19 & 0.042 & 0.211 & 14840 & . & 20 & 0.045 & 3.02 & 12790 & . & $21 *$ & 0.047 & 9.23 & 8880 \\
\hline 22 & 0.048 & 4.5 & 12370 & . & 23 & 0.049 & 4.57 & 18590 & . & 24 & 0.049 & 9.2 & 18550 \\
\hline 25 & 0.049 & 9.3 & 12770 & . & 26 & 0.057 & 0.28 & 13730 & . & 27 & 0.057 & 0.14 & 9750 \\
\hline 28 & 0.061 & 3.03 & 11650 & . & 29 & 0.062 & 0.61 & 9640 & . & $30 *$ & 0.067 & 920 & 13750 \\
\hline 31 & 0.067 & 9.2 & 11730 & . & 32 & 0.073 & 3.04 & 12210 & . & 33 & 0.086 & 0.43 & 12110 \\
\hline 34 & 0.088 & 0.49 & 8525 & . & 35 & 0.09 & 4.6 & 11010 & . & 36 & 0.10 & 9.3 & 7720 \\
\hline 37 & 0.10 & 6.9 & 8200 & . & $38 *$ & 0.10 & 0.49 & 8200 & . & 39 & 0.10 & 9.4 & 10750 \\
\hline 40 & 0.10 & 7.0 & 10750 & . & 41 & 0.10 & 7.1 & 16230 & . & 42 & 0.13 & 0.33 & 7560 \\
\hline 43 & 0.14 & 0.67 & 7600 & . & 44 & 0.14 & 0.98 & 7440 & . & 45 & 0.14 & 1.4 & 7330 \\
\hline 46 & 0.16 & 9.8 & 9580 & . & 47 & 0.19 & 38 & 2520 & . & 48 & 0.20 & 5.0 & 8720 \\
\hline 49 & 0.20 & 10.1 & 8760 & . & $50 *$ & 0.21 & 41 & 6270 & . & 51 & 0.26 & 10.3 & 8580 \\
\hline 52 & 0.34 & 0.84 & 5020 & . & 53 & 0.38 & 11.4 & 7120 & . & 54 & 0.40 & 42.2 & 1980 \\
\hline 55 & 0.47 & 1.2 & 4500 & . & 56 & 0.55 & 11.1 & 7140 & . & 57 & 0.6 & 1.5 & 5010 \\
\hline 58 & 0.89 & 2.2 & 4710 & . & 59 & 1.25 & 12.5 & 6470 & . & 60 & 2.7 & 12.5 & 4020 \\
\hline 61 & 5.5 & 13.7 & 4260 & . & & & . & & . & $62^{*}$ & 0.012 & $\infty$ & 15225 \\
\hline $63^{*}$ & 0.027 & $\infty$ & 11800 & . & 64 & 0.07 & $\infty$ & 11490 & . & 65 & 0.20 & $\infty$ & 8800 \\
\hline
\end{tabular}

is also compatible with $\epsilon_{P} / \epsilon_{V} \approx 1 / 3$, as measured in the stratosphere (Lindborg $\left.\mid 2006\right)$. In fact, such a quasi-equipartition of energy is found in a large range of wave-numbers, as shown in Marino et al. (2015b) in the forced case. It immediately implies that the Ellison scale $L_{E l l}$ goes as $L_{i n t} F r$, for which in fact the scaling is excellent (see Fig. 6p below), and that $L_{E l l} \sim L_{B} \sim u_{r m s} / N$. Note also that a pattern is discernible in $E_{P} / E_{V}$ with, on average, higher relative vertical velocity and higher relative potential energy at low Rossby number. As a function of Reynolds number, $r_{E}$ decreases on average for Re larger than $\approx 10^{4}$, because of the initial conditions (not shown).

The ratio of vertical to total kinetic energy $\left\langle w^{2} / 2\right\rangle / E_{V}$ is given in Fig. 1 (b). At low $F r, \mathcal{R}_{B}$, with weak nonlinear mode coupling, its high value for the runs of Table 1 is due to initial conditions, taken with a rough equipartition between velocity modes in all directions, in order to let anisotropy develop dynamically. A similar reduction in vertical velocity for rapidly rotating flows in the absence of stratification was observed in laboratory experiments (van Bokhoven et al. 2009), together with a weaker dissipation. In an intermediate range of parameters, around $F r \approx 0.1$, there is a small plateau, the 
TABle 2. Parameters for two other sets of DNS identified by Id and ordered, for each set, by their Froude number $F r$, with $R o$ and Re the Rossby and Reynolds numbers computed at the time of maximum of enstrophy for each run. $\mathcal{R}_{B}=R e F r^{2}$, and $n_{p}^{3}$ is the total number of grid points for each run. In runs $\mathbf{Z x}, x=[1,8]$, the initial conditions are large-scale isotropic and random for the velocity field, and zero for the temperature as for the runs of Table 1 . whereas in the $\mathbf{Q x}$ runs, $x=[9,17]$, initial conditions are in geostrophic balance for velocity and temperature fluctuations. For both sets, $N / f \approx 5$, a value close to what is found in the ocean.

\begin{tabular}{ccccccccccccc}
\hline Id & $n_{p}$ & $F r$ & $R o$ & $R e$ & $\mathcal{R}_{B}$ & . Id & $n_{p}$ & $F r$ & $R o$ & $R e$ & $\mathcal{R}_{B}$ \\
\hline Z1 & 256 & 0.042 & 0.208 & 3458 & 6.1 &. & $\mathbf{Z 2}$ & 512 & 0.063 & 0.316 & 6202 & 24.6 \\
$\mathbf{Z 3}$ & 256 & 0.064 & 0.321 & 3358 & 13.7 &. & $\mathbf{Z 4}$ & 512 & 0.064 & 0.321 & 6643 & 27.2 \\
$\mathbf{Z 5}$ & 128 & 0.065 & 0.325 & 1694 & 7.1 &. & $\mathbf{Z 6}$ & 256 & 0.097 & 0.487 & 2951 & 27.8 \\
$\mathbf{Z 7}$ & 256 & 0.651 & 3.255 & 1657 & 702 &. & $\mathbf{Z 8}$ & 256 & 3.296 & 16.481 & 1706 & 18578 \\
\hline \hline Q9 & 256 & 0.007 & 0.036 & 5221 & .25 &. & $\mathbf{Q 1 0}$ & 256 & 0.015 & 0.073 & 4973 & 1.1 \\
Q11 & 256 & 0.039 & 0.197 & 3706 & 5.6 &. & $\mathbf{Q 1 2}$ & 128 & 0.067 & 0.335 & 1617 & 7.2 \\
Q13 & 256 & 0.075 & 0.373 & 3130 & 17.6 &. & $\mathbf{Q 1 4}$ & 512 & 0.076 & 0.382 & 6278 & 36.3 \\
Q15 & 256 & 0.111 & 0.555 & 2537 & 31.2 &. & $\mathbf{Q 1 6}$ & 256 & 0.577 & 2.817 & 2003 & 667 \\
Q17 & 256 & 1.290 & 6.451 & 2008 & 3341 &. & & & & & & \\
\hline
\end{tabular}

stratification being strong enough to prevent most of the vertical motions; so,

$$
w_{r m s} \lesssim u_{r m s} \text {. }
$$

As turbulence strengthens with increasing $\mathrm{Fr}$, vertical motions develop slowly after that plateau, with an approximate scaling $\left\langle w^{2}\right\rangle \sim\left\langle u_{\perp}^{2}\right\rangle F r^{1 / 4}$. The origin of such a weak scaling is not clear, and no scaling is found in terms of $N / f$. We recall here that the vertical velocity $w \hat{e}_{z}$ is also a direct measure of wave activity since, in a wave-vortex decomposition as performed e.g. in Bartello (1995); Herbert et al. (2016), vortical modes have vanishing $w$. The presence of rotation facilitates vertical motions in the form of upward propagating inertial waves along Taylor columns that would form if there was no stratification, as clearly observed, including when the small scales develop strong vorticity (Davidson et al. 2006; Mininni et al. 2012): the vertical velocity is leaving a trace of the influence of rotation on the system and its increase is consistent with a wave-vortex analysis. The scaling in equation (3.2) may seem unexpected. Dimensional analysis using incompressibility at large scale would predict $w_{r m s} / u_{r m s} \sim F r$, ruled out by the data with isotropic ICs, but in agreement with the data with QG initial conditions at low Froude numbers, with this energy ratio reaching the value obtained with the isotropic ICs for $F r \approx 0.1$. We know that strong vertical velocities develop for intermediate $F r$ when the buoyancy and nonlinear terms balance each other, leading to a "saturation" vertical energy spectrum $E_{\|} \sim N^{2} k_{\|}^{-3}$. The model developed in Rorai et al. (2014), adding the buoyancy flux to a Vieillefosse description of intermittency for FD'T, leads to $E_{\|} \approx E_{P} \approx E_{V}$ under the hypothesis that the characteristic vertical scale is the buoyancy scale. In these intermittent regions identified by low Richardson numbers, high vertical velocities appear, due to strong turbulent stirring leading to overturning of layers. Note that it is argued in Maffioli \& Davidson (2016) that gradients are much larger in the vertical, or that $w$ is really at small scale. Thus, these authors advocate $\left\langle w^{2}\right\rangle \sim \epsilon_{V} / N$. With $\epsilon_{V} \sim \epsilon_{D} F r$ (see 3.3 below), this leads again to $w / u_{\perp} \sim F r$. On the other hand, in their paper, $\beta$ is viewed as a constant denoted $A_{k}<1$, independent of dimensionless parameters, so their estimate is rather $w / u_{\perp} \sim F r^{1 / 2}$, unlike our data at high $F r$. 

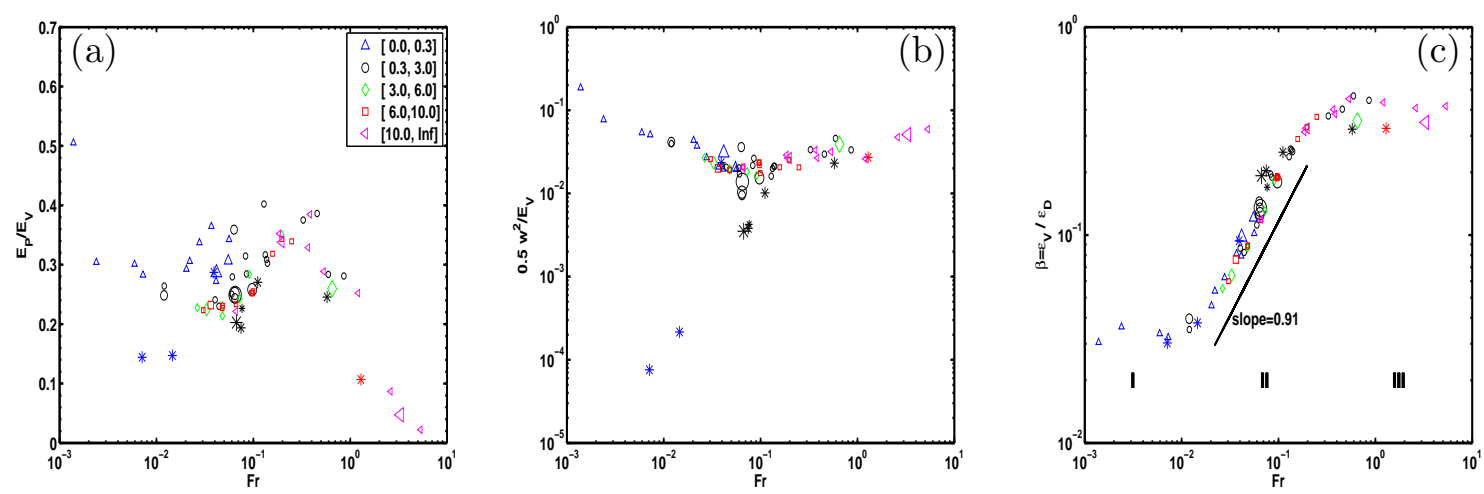

FiguRE 1. (Color online) Variation with Froude number of the ratio of potential to kinetic energy $(a)$, of vertical to kinetic energy $(b)$, and of the rate of kinetic energy dissipation compared to its dimensional evaluation, $\beta=\epsilon_{V} / \epsilon_{D}(c)$. Colors/symbols indicate binning in Rossby number for all runs: blue triangles for $0<R o \leqslant 0.3$, black circles for $0.3<R o \leqslant 2.9$, green diamonds for $2.9<R o \leqslant 6.0$, red squares for $6.0<R o \leqslant 10.0$, and magenta inverted triangles for $R o>10$ (see insert). The runs of Table 2 are indicated either by a star for those with quasi-geostrophic initial conditions ( $\mathrm{Q}$ runs), or by a hollow symbol for the runs with $\theta(t=0)=0$; their relative size is proportional to viscosity, thus inversely proportional to $R e$ and to numerical resolution. We define the three dynamical regimes as: I for strong waves $(F r \lesssim 0.01)$, II for eddy-wave interactions $(0.01 \lesssim F r \lesssim 0.2)$, and III for strong stratified turbulence $(F r \gtrsim 0.2)$, as indicated in (c); note the quasi-linear scaling of $\beta$ with $F r$ in regime II, namely $\beta \sim \widetilde{F r}^{0.91}$.

\subsection{Effective versus dimensional dissipation and the three regimes of $R S T$}

The dissipation efficiency of rotating stratified flows $\beta$ is shown in Fig. 11(c) as a function of $F r$; it clearly displays three regimes. For small $F r$ up to $F r \approx 0.01, \epsilon_{V}$ is low and constant. Similar low dissipation efficiency, of the order of a few percents, is obtained for runs corresponding to the Upper Troposphere and Lower Stratosphere region, with low Froude numbers, as analyzed for example in Paoli et al. (2014) using a sub-grid model. We find that, above $F r \approx 0.01, \beta$ grows quasi-linearly with $F r$, with a least-square fit giving a slope of 0.91 after which $\beta$ saturates, for $F r \gtrsim 0.2$. Thus,

$$
\beta=\epsilon_{V} / \epsilon_{D} \sim F r \quad[\text { Intermediate regime, } I I],
$$

thereby defining the three dynamical regimes of RST, namely I, II \& III, ordered by increasing $F r$. Such a scaling is also found when examining the small-scale energy flux in the forced case in the presence of an inverse cascade (Marino et al. 2015a). $\beta$ saturates at a value close to unity for highly turbulent flows at higher Froude numbers, as found as well in Maffioli \& Davidson (2016), although their values at peak of enstrophy are a bit higher. It may be related to the fact that they compute in boxes of small aspect ratio, between $1 / 4$ and $1 / 6$, a geometry that can favor vertical gradients and shear, and which can lead to a more active turbulence, as found in Mininni \& Pouquet (2017). When the turbulence strengthens, so does the direct energy cascade through baroclinic instability, frontogenesis and nonlinear coupling of eddies (McWilliams 2016). It should finally be noted that we do not expect actual transitional values of $\beta$, between regimes I \& II, and II \& III, to be similar for similar control parameters in different flow geometries, but we do expect the scaling $\beta \sim F r$ to hold, as shown here contrasting isotropic versus QG initial conditions (see also the dimensional argument in the Appendix showing how the transfer time to small scales is moderated by the stratification). 

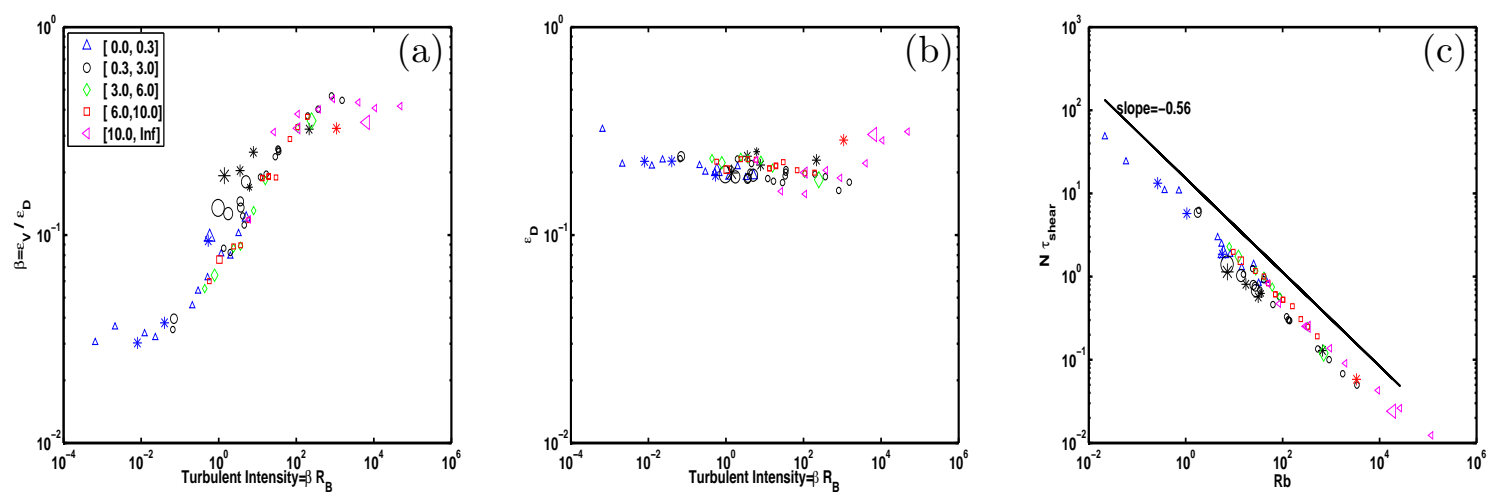

FiguRE 2. (Color online) Variation of $\beta(a)$ and $\epsilon_{D}=u_{r m s}^{3} / L_{\text {int }}$ (b) with turbulent intensity parameter $\mathcal{R}_{I}=\epsilon_{V} /\left[\nu N^{2}\right]$. In (c) is shown $R i^{1 / 2} \equiv N \tau_{\text {shear }}$ as a function of the buoyancy Reynolds number $\mathcal{R}_{B}=\beta^{-1} \mathcal{R}_{I}$, with $\tau_{\text {shear }}=\left\langle\partial_{z} u_{\perp}\right\rangle$. All plots have binning in Rossby number, and symbols are as described in the caption of Fig. 1. The scaling $N \tau_{\text {shear }} \approx \mathcal{R}_{B}^{-0.56}$ extends through regimes I \& II with lower $\mathcal{R}_{B}$ and $F r$, and possibly regime III.

If we now examine the variations of $\beta$ with $\mathcal{R}_{I}=\epsilon_{V} /\left[\nu N^{2}\right]$, we see in Fig. 2(a) that we again have good scaling throughout, with $\beta \sim \mathcal{R}_{I}^{1 / 3} \sim \beta^{1 / 3} \mathcal{R}_{B}^{1 / 3}$. It is easy to show that this is compatible with equation 3.3 , since $\beta \sim \mathcal{R}_{B}^{1 / 2} \sim F r$, omitting a dependency in $R e^{1 / 3}$, although it appears clearly in Fig. 2 that, at fixed $\mathcal{R}_{I}$ and lower $R e, \beta$ is measurably larger since $\mathrm{Fr}$ is larger. This indicates that care must be taken when interpreting data as a function of dimensionless parameters. In $\mathcal{R}_{B}$, the transitions between the three regimes occur respectively for $\approx 2$ and $\approx 200$. Such values are relevant for example in the ocean thermocline, where $\mathcal{R}_{B} \approx 10-100$ (Fleury \& Lueck 1994), as well as in lakes in which an average for $\mathcal{R}_{B}$ is $\approx 200$ (Bouffard \& Boegman 2013 ).

The dimensional kinetic energy dissipation $\epsilon_{D}$ (Fig. $2 \mathrm{~b}$ ) is constant across parameters, and across initial conditions, except for very small or large $F r$ values. A slight trend towards smaller values at higher $\mathcal{R}_{I}$, which can be attributed to smaller $r m s$ velocities, is discernible. Finally, we show in Fig. 2(c) that $R i^{1 / 2} \sim \mathcal{R}_{B}^{-1 / 2}$, a scaling compatible with $R i \sim \mathrm{Fr}^{-2}$ at constant Reynolds number. At low $F r$, this relation gives the strength of vertical gradients (slanted because of rotation), and at higher $F r$, it indicates a progressive return to isotropy and to only a single time-scale determining the dynamics, transfer and dissipation of such turbulent flows. Note that the three results in equations 3.1 3.3 may not be entirely new but, taken together, they define the key ingredients for establishing the scaling of the mixing efficiency which is discussed in 4 .

\section{Mixing and dissipation}

\subsection{Definitions of mixing efficiency and flux Richardson number}

Irreversible mixing is found in the laboratory to be triggered by merging KelvinHelmoltz billows (Patterson et al. 2006), highly unstable as Re increases. In the absence of rotation, parameter space has been separated into three regions in terms of $F r$ and Re (Luketina \& Imberger 1989): for small Re and Fr, waves are dominant and there are no turbulent motions, whereas for high Fr and Re, isotropic turbulence prevails. The intermediate region with roughly $F r \leqslant 1, \mathcal{R}_{B} \geqslant 10$ is where turbulence is anisotropic and strongly interacting with waves. The data on which these conclusions are based comes 

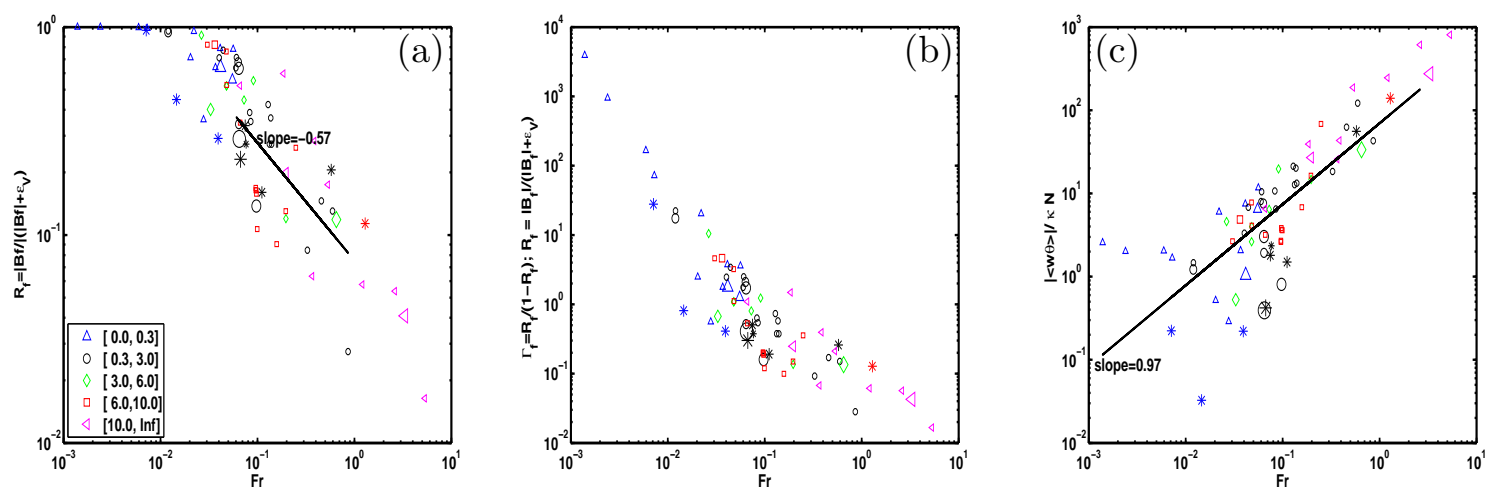

Figure 3. (Color online) Variation with Froude number of the flux Richardson number $R_{f}$ and of the mixing efficiency $\Gamma_{f}(a, b)$, both defined in equation 4.1 as well as of the effective diffusivity $\kappa_{\rho} / \kappa(c)$, with $\kappa_{\rho}=N^{-1}\langle w \theta\rangle$. Binning is performed in Ro (see Fig. 1 for symbols). A transition at $F r \approx 0.02$ is seen in all three plots. Scalings are given as indications.

from the analysis of turbulent plumes active in tidal estuary flows (Stillinger et al. 1983 ; Stacey et al. 1999). We find similar transitions with mild rotation, as shown in $\$ 3.3$.

In terms of the temporal evolution of vertical kinetic and potential energy density, one is led to compare the buoyancy flux $B_{f}=N\langle w \theta\rangle$ with the dissipation rates, the Coriolis force not affecting the energy balance. Performing space-averaging, one can write:

$$
D_{t} E_{V}=-B_{f}+\epsilon_{V}, \quad D_{t} E_{P}=B_{f}+\epsilon_{P} .
$$

In order to quantify the relative magnitudes of these terms, several expressions have been introduced in the literature. Concerning the momentum equation, one traditionally defines the flux Richardson number $R_{f}$ and its associated mixing efficiency $\Gamma_{f}$ as:

$$
R_{f}=\frac{B_{f}}{B_{f}+\epsilon_{V}} \quad, \quad \Gamma_{f}=\frac{R_{f}}{1-R_{f}}=\frac{B_{f}}{\epsilon_{V}} .
$$

The functional variation of $R_{f}$ with gradient Richardson number is central to numerical studies of geophysical flows. The mixing efficiency $\Gamma_{f}$ is singular for $R_{f}=1$, i.e. for fully mixed potential and kinetic modes (see Mashayek \& Peltier (2013); Salehipour \& Peltier (2015); Mashayek et al. (2017) for a discussion on the definitions of mixing efficiency). This corresponds to negligible kinetic energy dissipation, i.e. a limit of zero Froude number. As we shall see in Fig. $3 \Gamma_{f}$ does reach high values at low $F r$, in excess of $10^{3}$. Many recent works indicate variations with parameters as Re grows.

\subsection{Mixing and effective diffusivity as a function of parameters}

We evaluate $\Gamma_{f}$ at peak of dissipation, whereas in Stretch et al. (2010), it is computed as the ratio of two time integrals after more than $90 \%$ of the energy has dissipated. Since energy decay in turbulence is self-similar, these two methods should lead to comparable scalings. We show in Figs. 3(a,b) $R_{f}$ and $\Gamma_{f}$ as a function of Froude number. At low $F r$, $\epsilon_{V}$ is negligible compared to $B_{f}$, and $R_{f} \approx 1$. A sharp transition occurs for $F r \approx 0.02$, with $R_{f}$ decreasing continuously thereafter, and no visible saturation. The decline in $R_{f}$ begins in the intermediate range in which waves and vortices interact strongly. Similarly, $\Gamma_{f}$ shows a transition for $F r \approx 0.02$, with a change in slope from $\Gamma_{f} \approx F r^{-2}$ to $\approx F r^{-1}$, as $F r$ grows (approximate scalings), and with a variation of several orders of magnitude. At higher $F r$ (regimes II \& III), a power-law scaling seems likely, as for $R_{f}$. The variation 

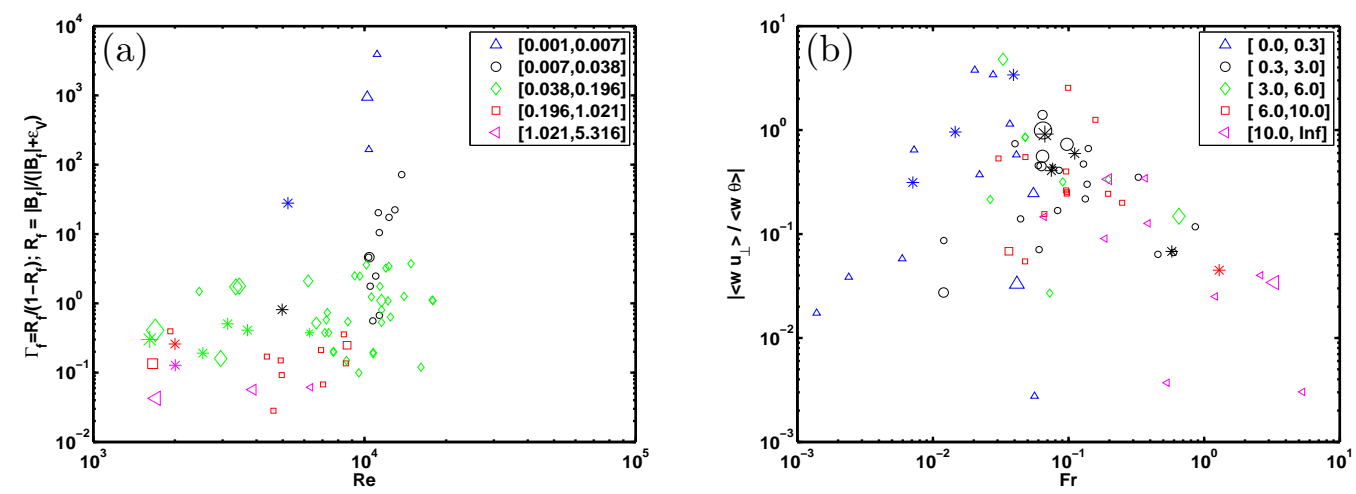

Figure 4. (Color online) (a): Variation of mixing efficiency $\Gamma_{f}$ with Reynolds number, with binning in Froude number (see insert). (b): Variation with $F r$ of the ratio of the vertical flux of horizontal velocity to the vertical flux of buoyancy, with binning in Rossby number. The size of symbols for both plots is described in the caption of Fig. 1.

of $R_{f}$ with Richardson number mirrors its variation in terms of $\mathrm{Fr}$ (not shown). Another example of strong variation of $\Gamma_{f}$ is found in Bluteau et al. $(2013)$ where $\Gamma_{f}=0.2$ is only valid in the range $7<\mathcal{R}_{B}<100$, whereas we find it for $100 \lesssim \mathcal{R}_{B} \lesssim 1000$.

The overall variation of $\Gamma_{f}$ with $F r$ and $\mathcal{R}_{B}$ is similar to that found in the absence of rotation but with shear (Mater \& Venayagamoorthy 2014). These authors further note that the centroid of such a curve depends on what flows are studied, as for example in the data of Lozovatsky \& Fernando (2013) where the centroid is shifted to higher $\mathcal{R}_{B}$. This presents a challenge, since parameterization schemes are mostly based on DNS, which may still be at too low a value of $\mathcal{R}_{B}$, and since using $\mathcal{R}_{B}$ implies studying variations in terms of both stratification (through $F r$ ) and of turbulence (through $R e$ ). The variations with Reynolds and buoyancy Reynolds numbers are discussed further in 77

In Fig. 3(c) we plot the effective diffusivity $\kappa_{\rho}$, relative to the molecular diffusivity $\kappa$. It is proportional to the buoyancy flux $B_{f}$. Taking the notation in Ivey et al. (2008):

$$
\kappa_{\rho}=B_{f} / N^{2}, \quad \kappa_{\rho} / \kappa=\langle w \theta\rangle /[N \kappa] ;
$$

$\kappa_{\rho}$ is comparable to $\kappa$ at low $F r$, and its increase with $F r$ is close to a linear variation, a least-square fit giving $\kappa_{\rho} / \kappa \approx F r^{0.97}$. Finally, a saturation begins to occur at high $F r$. This behavior can be interpreted as being due to an increase in buoyancy flux because of more vigorous stirring when $\mathcal{R}_{B}$ increases, at relatively constant $R e$. When comparing the high- $F r$ values of $\kappa_{\rho} / \kappa$ to the model proposed in Barry et al. (2001), a rough agreement is obtained. Similarly, when examining a compilation of observational data for both salinity and temperature in the ocean together with numerical data for purely stratified flows, it is found in Bouffard \& Boegman (2013) that a transition occurs in $\kappa_{\rho}$, at $\mathcal{R}_{B} \approx 100$, between a $\mathcal{R}_{B}^{3 / 2}$ and a $\mathcal{R}_{B}^{1 / 2}$ scaling (see also Shih et al. $(2005)$ for the latter). This enhancement of dissipation and of transport coefficients, such as anomalous diffusivity, is expected in turbulent flows as shown in numerous studies of FDT (Ishihara et al. 2009), as well as in the strongly stratified case (Ivey et al. 2008). In this latter instance, it is probably due to strong intermittency, a signature of strongly stably stratified flows (Rorai et al. 2014), such as in the planetary boundary layer (Finnigan 1999), or in the ocean (D'Asaro et al. 2011).

We show in Fig. 4(a) the mixing efficiency $\Gamma_{f}$ as a function of Reynolds number. 

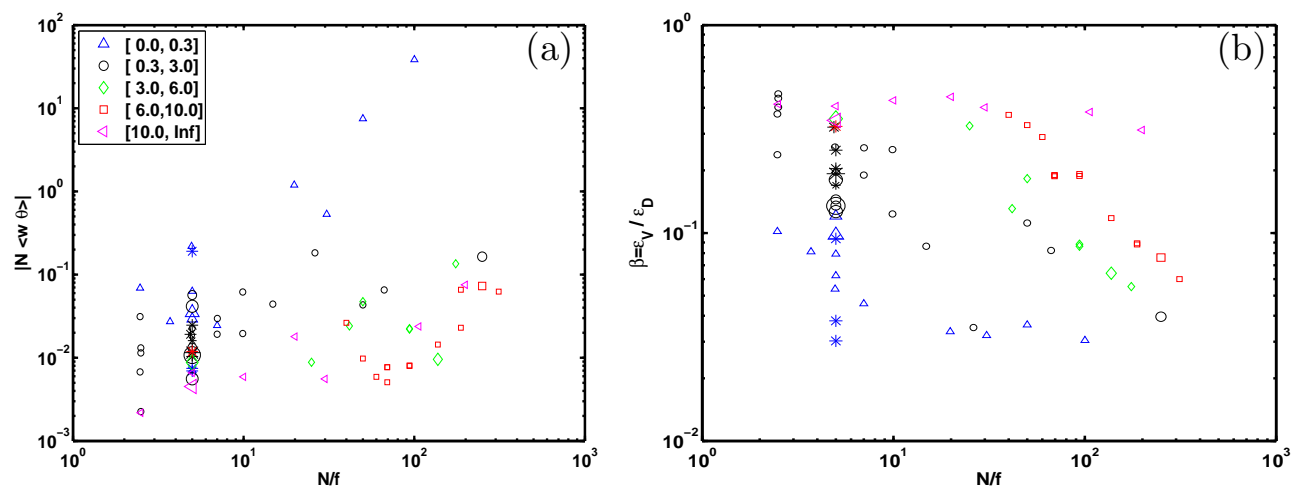

Figure 5. (Color online) Variation with $N / f=R o / F r$ of the buoyancy flux $B_{f}=\langle N w \theta\rangle(a)$, and of the dissipation efficiency $\beta=\epsilon_{V} / \epsilon_{D}(b)$, with binning in $R o$ (see insert); symbols are as in Fig. 1. For a given $N / f$, both can take a large range of values.

Around $R e \approx 10^{4}$, which covers many of the runs of Table $1, \Gamma_{f}$ takes on a variety of values corresponding to how strongly the flow is stratified, with variations in excess of 1000. However, at lower Re (for most of the runs of Table 2), $\Gamma_{f}$ remains at lower values, the peak in $\Gamma_{f}$ being linked to the development of small-scale instabilities as $R e$ and $\mathcal{R}_{B}$ grow. Finally, another measure of the small-scale mixing efficiency of a flow is the ratio of the vertical fluxes of horizontal velocity to that of temperature fluctuations, as analyzed in Zilitinkevich et al. (2013). It is shown in Fig. 4(b) as a function of Fr; no clear scaling emerges although one could advocate a $\mathrm{Fr}^{-1}$ decrease for regimes II and III. Also, this ratio appears to be higher in the intermediate regime, on average.

\section{The combined roles of rotation and stratification}

The addition of rotation leads to the propagation of inertia-gravity waves whose dispersion relation depends on $N / f$. Thus, the atmosphere, with $N / f \sim 100$, and the ocean where $N / f \lesssim 10$ may differ in their statistical properties. For all runs of this paper, $N / f \geqslant 2.5$, so that stratification dominates. It is thus not surprising that the classical picture of mixing in stratified flows has not been changed in a significant way when weak rotation is included but in the absence of scale separation and of forcing. We do see an effect of rotation on the magnitude of the potential energy (see e.g. Fig. 17), strong rotation altering the large scales where the energy is contained. It was shown in Marino et al. (2015a) that rotation and stratification play complementary roles in the relative strength of the direct and inverse constant-flux energy cascades in the forced case: the small-scale cascade is weaker the smaller the Froude number, and conversely the largescale cascade is stronger the smaller $R o$ is, in both cases affecting the effective dissipation of energy in the small scales and thus, presumably, the mixing properties of such flows. For the flows of Table 1 all micro Rossby numbers, $R_{\omega}=\omega_{r m s} / f$, are larger than 3.1, with $R o \geqslant 0.11$. Thus, the small-scale vorticity created by the nonlinear dynamics of the flow, including in the presence of strong waves, is dominant at small scale, compared to the imposed rotation; note that such values for $R_{\omega}$ are plausible for geophysical flows.

Figs. $5(\mathrm{a}, \mathrm{b})$ give the variations with $N / f$ of the buoyancy flux $B_{f}$ and of the dissipation efficiency $\beta$. At a given $N / f$, there is less buoyancy flux the higher the Rossby number, and for a given bin in $R o, B_{f}$ is larger the higher $N / f$ : as the Froude number increases, 

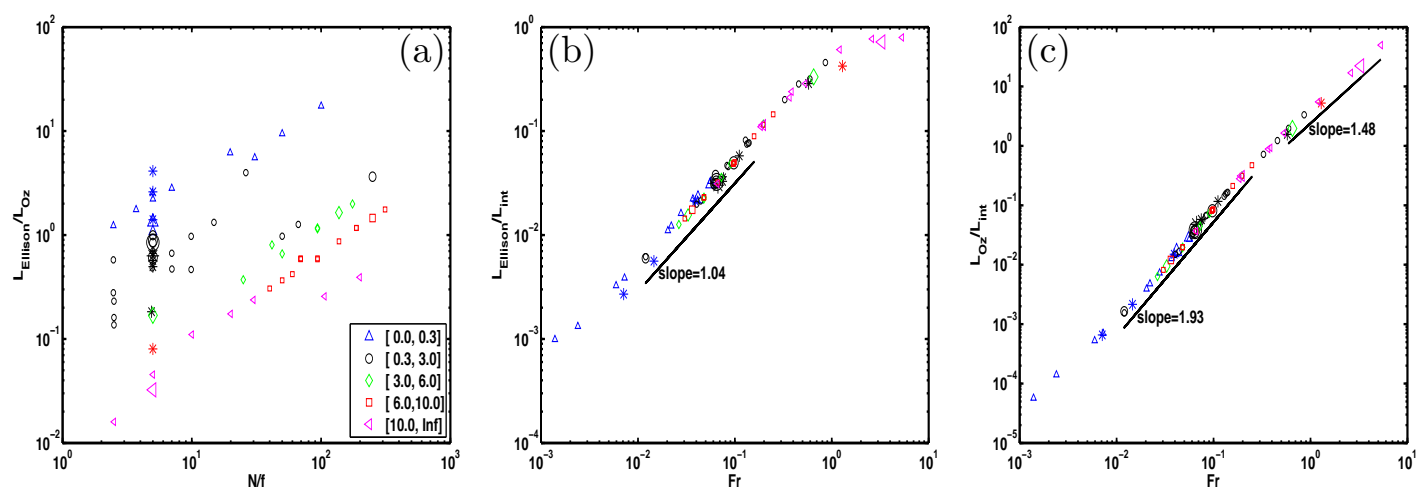

Figure 6. (Color online) Ellison scale $L_{E l l}=\theta_{r m s} / N$ relative to the Ozmidov scale $\ell_{O z}(a)$ or the integral scale $L_{\text {int }}(b)$, as a function of $N / f(a)$, and of $F r(b)$. In (c) is given $\ell_{O z} / L_{\text {int }}$ as a function of $F r$. All plots have binning in Ro (and see caption of Fig. 1). Note the small dispersion in the scaling of length scales versus Froude number (approximate scalings are given).

the buoyancy flux decreases. The efficiency of dissipation is higher the higher the Rossby number (Fig. 5b), again at fixed $N / f$. Perhaps the scaling of $\beta$ with $F r$ is somewhat modified by rotation, leading to the slightly sub-linear law observed in Fig. 1(c): there may be less direct energy transfer for strong rotation, as is observed in the forced case (Marino et al. 2015a), and thus it takes a higher Froude number to reach a given level of dissipation. A clear effect of the Rossby number on dissipation in RST was also shown in the forced case in Pouquet et al. (2017).

Thus, the absence in the overall statistical properties of clear scaling in Ro simply shows that the energy transfer to the small scales is dominated by a combination of stratification and turbulence. However, the primary purpose of this study is not to examine the role of rotation directly. Such a study would be helped by analyzing flows with $N / f<1$, a parameter range which is relevant neither to the atmosphere nor to the ocean. For $N / f>1$, the most important role of rotation in such flows is the triggering of an inverse cascade of energy to the large scales, attenuating the energy transfer to the small scales (Pouquet \& Marino 2013, Marino et al. 2015a), Pouquet et al. 2017 ). In the absence of forcing and of scale separation, such an inverse cascade cannot develop in general.

\section{The Ellison scale and a generalized mixing efficiency}

Similarly to characteristic time scales, one can also examine characteristic length scales. A comparison of the Thorpe and Ellison scales in stratified turbulence was performed in Mater et al. (2013) (see also Dillon (1982)). The Thorpe scale $L_{T}$ corresponds to the vertical distance a parcel of fluid must be moved to produce a stable density profile, suppressing inversions, and as such gives an idea of the size of local mixing structures in the fluid; it was computed as a function of $\mathcal{R}_{B}$ for the purely stratified case in Métais \& Herring (1989). In Mater et al. (2013), $L_{T}$ is found to be strongly linearly correlated with the Ellison scale $L_{E l l}$. Furthermore, the Thorpe length normalized by the Ozmidov 
scale is found to vary as $\mathrm{Fr}^{-3 / 2}$ for $\mathrm{Fr}>1$ and as $\mathrm{Fr}^{-1 / 2}$ for $\mathrm{Fr}<1$, the latter with an excellent scaling for $0.05 \leqslant F r \leqslant 0.3$. Thus, $L_{T} \sim L_{B}$ for $F r<1$, whereas $L_{T} \sim L_{\text {int }}$ for $F r>1$. In the latter case, stratification is weak and structures in density follow the integral length scale, whereas in the former case of strong stratification, density changes occur over the vertical layer width, i.e. the buoyancy scale. Note that this also implies that, at small Froude number, $L_{T} / L_{\text {int }} \sim F r$ and that $E_{P} \sim E_{V}$ (Mater et al. 2013).

In Fig. 6 are shown the variations of $L_{E l l}$ normalized by the Ozmidov scale (a) and integral scale (b), as a function of $N / f$ (a), and of $F r(b)$, with binning in Rossby number. We conclude that the Ellison scale is larger, the larger $F r$ is, as expected. As a function of Froude number, $L_{E l l} / \ell_{O z}$ decreases, in a linear fashion for the intermediate regime, and with little dispersion among the runs (not shown). One could argue that with $\ell_{O z}=\left[\epsilon_{V} / N^{3}\right]^{1 / 2}$, for small Froude number, $\ell_{O z} / L_{i n t} \sim F r^{2}$ in the intermediate regime in which $\beta \sim F r$, whereas for high $F r, \ell_{O z} / L_{\text {int }} \sim F r^{3 / 2}$, in rough agreement with scaling laws, as indicated in Fig. 6(c) giving $\ell_{O z} / L_{i n t}=f(F r)$ with least-square fits of respectively 1.93 and 1.48 , the transitions taking place for $F r \approx 0.01$ and $F r \approx 0.2$, in agreement with the transitions for $\beta$ (see Fig. 1k).

On the other hand, the linear variation $L_{E l l} / L_{\text {int }} \sim F r$ in Fig. 6(b), with a leastsquare fit giving $\sim F r^{1.04}$, is a direct consequence of the scaling law $\theta_{r m s} \sim u_{r m s}$. The only transition in this power-law behavior takes place for $F r=\mathcal{O}(1)$, in which case $L_{E l l} \lesssim L_{i n t}$, with $L_{E l l}$ remaining smaller than $L_{\text {int }}$ because of the $1 / k$ factor in the definition of $L_{i n t}$. There is also an indication of a slight saturation at low $F r$.

Another measure of the relative importance of terms in the Boussinesq equations is defined through the ratio of the two dissipative terms for momentum and temperature, which can differ even at unit Prandtl number. We use the following definitions (see also Osborn (1980); Venayagamoorthy \& Koseff (2016)):

$$
R_{f}^{*}=\frac{\epsilon_{P}}{\epsilon_{T}} \quad, \quad \Gamma_{f}^{*}=\frac{R_{f}^{*}}{1-R_{f}^{*}}=\frac{\epsilon_{P}}{\epsilon_{V}},
$$

with $\epsilon_{T}=\epsilon_{V}+\epsilon_{P}$ already defined in equation $(2.4) . \Gamma_{f}^{*}$, called the irreversible mixing efficiency in Mater \& Venayagamoorthy (2014), relates to the partition of energy between the kinetic and potential modes, i.e. to the importance of the waves versus nonlinear eddies at small scales. $R_{f}^{*}$ is shown in Fig. 7(a) as a function of $R i$. In the first regime of strong waves $(R i>1)$, it is small since the influence of initial conditions prevails, and similarly for the low $R i$ regime in which the potential-kinetic exchanges are inefficient, leading to an abrupt decrease in $R_{f}^{*}$. For intermediate values of $R i$, almost from $10^{-3}$ to roughly $10, R_{f}^{*}$ stays rather constant in a range between 0.3 and 0.4 . Note also that this data is consistent with the variations of $\epsilon_{V}$ and $\epsilon_{P}$ with $F r$ studied in Sozza et al. (2015) in a thin layer box.

We finally examine in Fig. $7(\mathrm{~b})$ the variations of $\Gamma_{f}^{*}$ with $R i f^{2} / N^{2}=\left[\tau_{\text {shear }} f\right]^{2}$, thus combining the effects of stratification and rotation. A rather small variation of $\Gamma_{*} \approx 0.5$ is obtained, with the low points corresponding to small $f$. There is a slow decline for small $R o$ and large $R i$ which may be related to sensitivity to initial conditions at low Fr: in regime I, $\epsilon_{V} \approx \epsilon_{P}$ is compatible with $E_{P} \approx E_{V}$ since, when the waves are strong to moderate, there is little nonlinear transfer and the dissipation is mostly contained in the large scales. Also, the highest value of $\Gamma_{f}^{*} \approx 0.7$ for $F r \approx 0.01$, corresponds obviously to flows with comparable kinetic and potential energy dissipation. This is likely associated in that regime to strong waves and intermittent bursts which are due to wave breaking which temporarily relax the flow to a quasi-equipartition of kinetic and potential energies across a wide range of scales, the more so the smaller the scale, as observed for example 

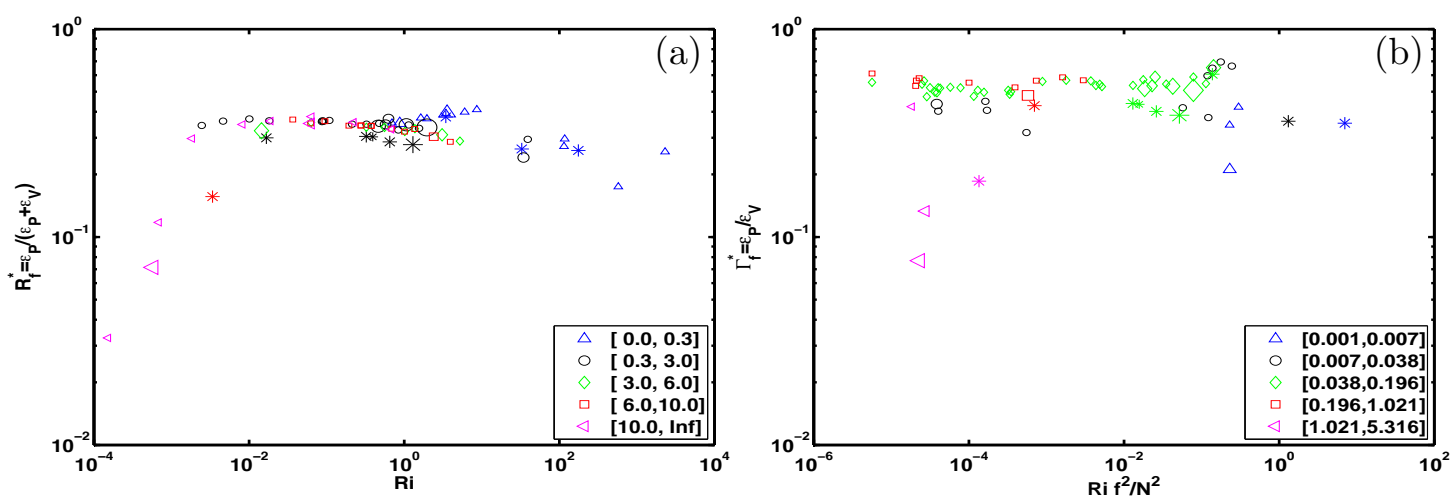

Figure 7. (Color online) Potential energy dissipation normalized by total dissipation, $R_{f}^{*}=\epsilon_{P} / \epsilon_{T}$, vs. Richardson number $R i(a)$, and $\Gamma_{f}^{*}=\epsilon_{P} / \epsilon_{V}$ vs. Rif $f^{2} / N^{2}=\left[\tau_{s h e a r} f\right]^{2}$ (b). Binning is performed in $R o$ in (a) and in $F r$ in (b) (see inserts, and caption of Fig. 1).

in Rosenberg et al. (2015). Our results corroborate those of Venayagamoorthy \& Koseff (2016): different measures of mixing, such as $\Gamma_{f}$ or $\Gamma_{f}^{*}$, give rather equivalent information, although it is not clear if this result will persist in the presence of forcing, at much higher buoyancy Reynolds numbers.

\section{Role of Reynolds number}

The variation of the intensity of the turbulence in rotating stratified flows can be measured by the Reynolds number, as well as by the buoyancy Reynolds number. Having high-enough $R e$ and $\mathcal{R}_{B}$ is known to be important for turbulent flows, to allow for coherent structures to develop including in the presence of strong stratification (see e.g. Laval et al. (2003)). However, from a numerical point of view, having high $R e, \mathcal{R}_{B}$ for low $\mathrm{Fr}$ is quite challenging and remains a goal for the near future. When taking the data for the runs of Tables 1 and 2 for a possible scaling of the dissipation efficiency $\beta$ with $\mathcal{R}_{B}$, we observe some scatter (see Fig. 8a). Specifically, we see that in the intermediate regime $\left(\mathcal{R}_{B}\right.$ between 10 and a few 100$)$, at fixed $\mathcal{R}_{B}$, there is a measurable variation in $\beta$, by contrast to regimes I, and to a lesser extent regime III. This scatter in regime II is larger than when examining variations with the Froude number itself, irrespective of the rotation (see Fig. 1(c)). In Fig. 8b, we see that overall, there is markedly less scatter when plotting $\beta$ as a function of the parameter $\left[N T_{L}\right]^{-2}$ as discussed in Mater \& Venayagamoorthy (2014) (see the Appendix, \$9.1); $T_{L} \equiv E_{V} / \epsilon_{V}$ is the effective kinetic energy transfer time. Expressing $\epsilon_{V}=\beta \epsilon_{D}$, we see that $N T_{L}=[\beta F r]^{-1}$; thus, the choice of the abscissa in Fig. 8(b) is to be able to make a direct comparison with $\mathcal{R}_{B}$ which scales as $\mathrm{Fr}^{2}$ at fixed $R e$. We also note that the regime transitions in terms of Richardson number occur for $R i \approx 0.1$ and $R i \approx 10$ (not shown).

The difference in data points scatter between Fig. 8(a,b) can be attributed to the variations with Reynolds number, as displayed in Figure 8(c). It shows clearly that the Reynolds number alone does not allow for predicting the effectiveness of dissipation, and consequently that of mixing efficiency, with a wide scattering of data points for $\beta$, irrespective of the initial conditions tested in this paper. However, we note that at a given Re, QG initial conditions lead to a substantially lower dissipation efficiency. We further note that Reynolds numbers are still quite low for these runs, when comparing 

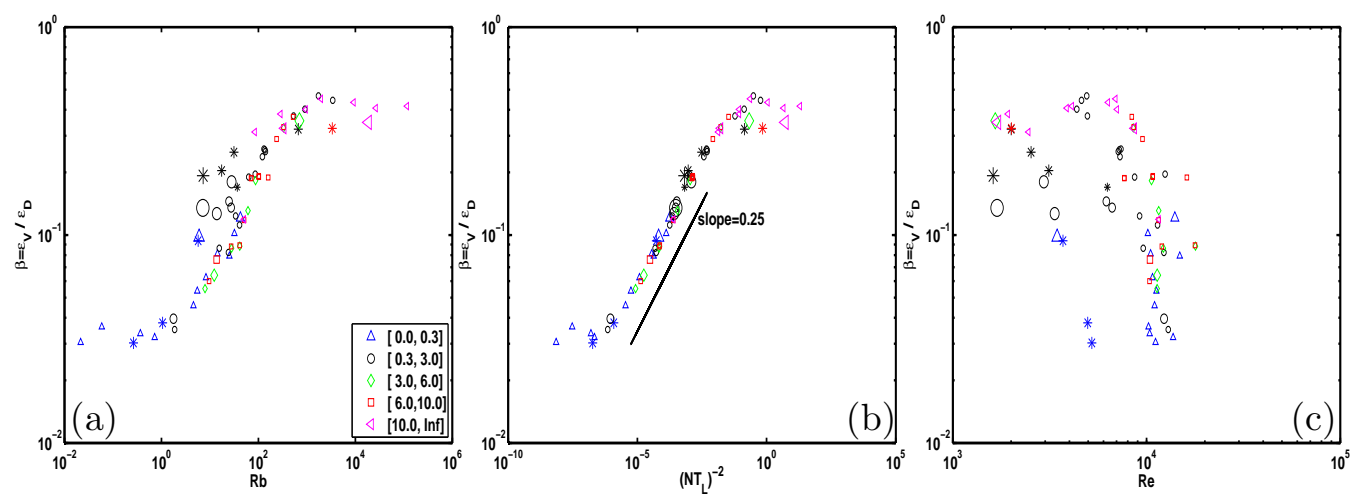

FiguRE 8. (Color online) Dissipation efficiency $\beta$ as a function of (a) $\mathcal{R}_{B}$, (b) $\left[N T_{L}\right]^{-2}$, and (c) $R e$ (see 9.1 for a discussion of $\left.N T_{L}=N E_{V} / \epsilon_{V}\right)$. Note the scaling $\beta \sim\left[N T_{L}\right]^{-1 / 2}$ in (b).

with geophysical flows. Similar conclusions can be drawn for the variation with $R e$ of the ratio of the Ellison scale normalized by the integral scale (not shown).

\section{Discussion, conclusion and perspectives}

A parametric study of mildly rotating stratified turbulence without forcing leads to a rather systematic quantitative assessment of its mixing and dissipative properties which depend on the Froude number provided the Reynolds number is high enough. Three different regimes are observed, in agreement with previous studies of purely stratified flows. These regimes are also identifiable in terms of the interaction parameter $\mathcal{R}_{I}$. The three basic laws illustrated in Fig. 1 are compatible with an intermediate regime characterized by the dynamics of waves and eddies interacting nonlinearly weakly, even though the full weak turbulence formalism leading to a set of closed integro-differential equations in terms of energy spectra cannot work for stratified flows because of a Froude number in the vertical of order unity (Billant \& Chomaz 2001). It is thus somewhat remarkable that the simple phenomenology embodied in the parameter $\beta \sim F r$, i.e. the efficiency of the turbulent dissipation, based on a ratio of characteristic time scales (see equation (9.4), may still apply on average for such flows.

Together with $\theta_{r m s} \sim u_{r m s}$ and a scaling for $w / u_{\perp}$ going as a quasi-constant at intermediate $F r$, these laws imply that the mixing efficiency $\Gamma_{f} \sim F r^{-2}$ as soon as $F r>$ 0.01 , and $\Gamma_{f} \sim F r^{-1} \sim \mathcal{R}_{B}^{-1 / 2}$ for $F r \lesssim \mathcal{O}(1)$. We emphasize that the actual values of the control parameter for the change of regimes may depend on the geometry and topology of such flows. In the intermediate regime, $\beta \sim F r$, showing the connection between buoyancy flux and nonlinear transfer leading to dissipation, with $\Gamma_{f} \beta^{2} \sim 1$. Note that, with $\mathcal{R}_{I} \sim F r \mathcal{R}_{B}$, this scaling law in the intermediate regime differs when expressed using $\mathcal{R}_{I}$. Finally the mixing efficiency measured in terms of the ratio of potential to kinetic energy dissipation, is shown to be rather constant. The scaling $\Gamma_{f} \sim F^{-1} \sim \mathcal{R}_{B}^{-1 / 2}$, in regime III at high $F r$ and $\mathcal{R}_{B}$, simply stems from the decoupling of the velocity and temperature, together with $\beta \approx 1$, leading to $B_{f} \sim N$.

Note also that $\Gamma_{f}$ and $R_{f}$ seem to be more sensitive to parameters with a clear indication of the three physical regimes in terms of $F r, \mathcal{R}_{B}$ or $\mathcal{R}_{I}$, than either $\left\langle w u_{\perp}\right\rangle /\langle w \theta\rangle$ or $E_{P} / E_{V}$. Furthermore, if $w^{2} / u_{\perp}^{2} \sim F r$ as advocated in Maffioli \& Davidson (2016), then the phenomenological arguments developed in our paper lead straightforwardly to 
$\Gamma_{f} \sim \mathcal{R}_{B}^{-3 / 4}$, which cannot be entirely ruled out given the scatter in data points for $\Gamma_{f}$, although it is not compatible with the data of Fig. 1 (b) with $w_{r m s} / u_{r m s} \sim 1$. We verified that taking as initial conditions geostrophically balanced flows did not alter our conclusions; similarly, having non-zero potential energy, but still unbalanced and with $w \neq 0$ ICs, we obtained the same fundamental scalings.

Local variations in Richardson number may trigger local density micro-structures, as observed in the ocean (Phillips 1972, Peltier \& Caulfield 2003). If the agreement of our results, without shear but with rotation, with previous results mainly for sheared purely stratified flows is striking, it remains to be seen whether it will persist in the presence of forcing, i.e. in the presence of a strong inverse cascade. We note that Waite \& Bartello (2006) already observed three regimes in the presence of forcing, with a switch for the energy cascade from predominantly inverse to direct.

Within the confines of the present parametric study with a wide range of buoyancy Reynolds numbers, $\Gamma_{f}$ is in fact rather variable, as in the purely stratified case. Rotation is essential for the existence of a dual constant-flux cascade of energy, implying twodimensional (horizontal) lateral mixing as well as vertical. If such mixing occurs in proportion to the ratio of the inverse to direct cascade, it will scale as $[R o F r]^{-1}$ (Marino et al. 2015a). In the absence of forcing, with large-scale initial conditions and with rotation weaker than stratification, all effects associated with the presence of rotation are severely quenched. As discussed in Mashayek \& Peltier (2013), shear can induce vortex pairing at moderate Reynolds number, reinforcing the potential for an inverse cascade in the presence of rotation but, on the other hand, as Re increases, 3D instabilities take over and shearing leads to enhanced dissipation.

Another issue, when incorporating rotation or stratification, will be to consider the role of anisotropy on statistics, spectra and structures, the role of nonlocal interactions among scales, and the role of potential vorticity $P_{V}$ and the magnitude of its nonlinear part; these will be the topic of future work. Several other extensions of the present study are desirable. On the one hand, a larger scanning in terms of Reynolds numbers is needed, but only feasible today at values comparable to or lower than what is presently achieved in this paper, without using modeling such as eddy viscosity or hyper-viscosity, or some other partial truncation of modes such as computing in boxes with small aspect ratio. From the numerical standpoint, the condition $\ell_{O z}>>\eta, \mathcal{R}_{I}>>1$ for strong and stratified turbulence to develop is hard to fulfill even with resolutions allowed by high-performance computing using available present-day technology. For example, in de Bruyn Kops (2015), the highest buoyancy Reynolds number that is reached, on a grid of $8192^{2} X 4096$ points, is $\approx 220$, still quite low compared to atmospheric and oceanic values (see also (Iyer et al. 2017) for a FDT run on a grid of $8192^{3}$ points with a Taylor Reynolds number of 1300). In the context of the turbulent planetary boundary layer (see e.g. Sukoriansky et al. (2005)), one can write simplified expressions for vertical mixing, governed by vertical velocity, and horizontal mixing on the basis of a return to isotropy model; this leads to agreement between these models, and laboratory and atmospheric data. One can also model the time-evolution of a mixing event by following characteristic length scales (Smyth et al. 2001).

For the oceans, the collapsing of mixing efficiency at high $\mathcal{R}_{I, B}$ embodied in the $\Gamma_{f} \sim$ $\mathcal{R}_{B}^{-1 / 2}$ scaling, might imply the lessening of water mass motions in the ocean, by at least a factor of 2 as found for the Antarctic Bottom Water (de Lavergne et al. 2016). The tide impinging upon oceanic bottom topography leads to the formation of small scales which, beyond the Ozmidov scale, become isotropic. This could imply that the strong dissipation and mixing which is observed, for example at the Hawaian ridge (Klymak et al. 2008), is 
propagating upward, in particular at mid latitudes where rotation plays a role, to scales of the order of $1 \mathrm{~km}$. This may lead to exchanges of light and dense waters and abyssal sinking (Ferrari et al. 2016), thereby affecting the net circulation patterns of the ocean. Thus, a better understanding of the dynamics of rotating stratified turbulence, and of the scaling of its mixing and dissipative properties with control parameters, may lead to better parametrization schemes to model more accurately these global phenomena.

Acknowledgments: AP is thankful to LASP and Bob Ergun for support, and to Colm Caulfied for a useful discussion while at IPAM. RM acknowledges support from the PRESTIGE program coordinated by Campus France (co-financed under Marie Curie FP7 PCOFUND-GA-2013-609102) and the PALSE program at the University of Lyon. Computations were performed at the National Center for Atmospheric Research, through an ASD allocation, as well as a new (2017) allocation of background time. NCAR is supported by the National Science Foundation. Finally, we also acknowledge requests by the reviewers to perform more runs (see Table 2), as well as to simplify the text.

\section{Appendices}

Many parameters and characteristic time-scales and length scales have been defined in the literature for rotating stratified turbulence, and we regroup some of them here for completeness. They allow for the definition of slightly different dimensionless parameters for which we also give an overview.

\subsection{Appendix A: Characteristic time scales}

The four global control parameters of the Boussinesq equations written in $\$ 2$ can be written as the ratio of large-scale characteristic times, namely:

$$
R e=\frac{\tau_{d i s s}}{\tau_{N L}}, R o=\frac{\tau_{w r}}{\tau_{N L}}, F r=\frac{\tau_{w g}}{\tau_{N L}},
$$

with $\tau_{\text {diss }}=L_{\text {int }}^{2} / \nu, \tau_{N L}=L_{\text {int }} / u_{r m s}, \tau_{w g}=1 / N$ and $\tau_{w r}=1 / f$ respectively the dissipation and eddy turn-over times, and the gravity and inertial wave periods; finally, $\operatorname{Pr}=\tau_{\kappa} / \tau_{\text {diss }}$ with $\tau_{\kappa}=L_{\text {int }}^{2} / \kappa$. The integral scale $L_{\text {int }}$ was defined in \$2, When linearizing the Boussinesq equations, one obtains inertia-gravity modes of frequency $\omega_{k}= \pm \sqrt{N^{2} k_{\perp}^{2}+f^{2} k_{\|}^{2}} / k$, with $k_{\|, \perp}$ referring to the vertical and horizontal directions (see e.g. Bartello (1995)). However, for the sake of simplicity, we define the above parameters using isotropy, i.e. omitting $\left[k_{\perp}, k_{\|}\right]$factors which would appear through the dispersion relation. One can define an effective transfer time for the kinetic energy, measured directly from observational or numerical data, as:

$$
T_{L} \equiv E_{V} / \epsilon_{V},
$$

whereas $\tau_{N L}=E_{V} / \epsilon_{D}=\beta T_{L}$ is based on a-priori large-scale characteristics of the flow, with $\beta=\epsilon_{V} / \epsilon_{D}$ as defined in equation (2.5).

In the absence of imposed shear, the Richardson number is based on a shear time $\tau_{\text {shear }}$ built from the vertical gradient of the mean horizontal wind $u_{\perp}$ :

$$
\tau_{\text {shear }}=1 /\left\langle\partial_{z} u_{\perp}\right\rangle, \quad R i=\left[N \tau_{\text {shear }}\right]^{2} .
$$

As such, $R i$ can be viewed as measuring the strength, in terms of time-scales, of the formation of internal turbulent shear layers due to nonlinear interactions to that of the vertical layers due to the gravity waves, omitting the effect of rotation. 
In the presence of several characteristic time and length scales, dimensional analysis is undetermined, even without rotation and for a unit Prandtl number. One particular set of parameters has been proposed in Mater \& Venayagamoorthy (2014), and we now analyze it, with some variation in notation. The crucial point is to emphasize the difference between the effective kinetic energy dissipation rate, $\epsilon_{V}$, and its dimensional evaluation, $\epsilon_{D}$, through their ratio $\beta=\epsilon_{V} / \epsilon_{D}$.

It is traditional in wave turbulence (Zakharov et al. 1992) to model the weaker transfer of energy to small scales due to the waves, when compared to a fully turbulent flow, by introducing a transfer time written a priori on dimensional grounds as:

$$
\tau_{t r} \equiv \tau_{N L} \frac{\tau_{N L}}{\tau_{w g}}=\frac{\tau_{N L}}{F r},
$$

using the small parameter adequate for the problem at small scale, here $F r<<1$. Thus, $\tau_{t r}>\tau_{N L}$, as expected. In the purely rotating case, one would use $\tau_{w r}=\tau_{N L} R o$ (Cambon \& Jacquin 1989).

It is then deduced that consistency between the two definitions of a transfer time, namely taking $T_{L}$ and $\tau_{t r}$ to be proportional, immediately implies that one must have:

$$
\beta \sim F r
$$

in the intermediate range. This scaling, confirmed by numerical data (Fig. 1. extended to $F r=1$; then, $\tau_{t r}=\tau_{N L}$ : the energy is transferred to small scales in an eddy turn-over time, the hallmark of FDT.

Note that a second characteristic time was also introduced in Mater \& Venayagamoorthy (2014), based again on $\epsilon_{V}$, and now on viscosity, namely:

$$
T_{\lambda}=\left[\nu / \epsilon_{V}\right]^{1 / 2}=\tau_{N L} /[\beta R e]^{1 / 2} .
$$

The dependence of $T_{\lambda}$ on $\sqrt{\nu}$ indicates that this time is linked to the Taylor scale $\lambda_{V}=$ $u_{r m s} / \omega_{r m s}=\sqrt{u_{r m s}^{2} \nu / \epsilon_{V}}$, by writing $\lambda_{V}=u_{r m s} T_{\lambda}$. Other relevant scales are discussed in Barry et al. (2001); Davis \& Monismith (2011); Mater \& Venayagamoorthy (2014).

\subsection{Appendix B: A note on dimensional analysis}

Control parameters can be defined using the characteristic times mentioned above:

$$
\begin{aligned}
& R_{g} \equiv\left[\tau_{t r} / T_{\lambda}\right]^{2}=u_{r m s}^{4} /\left[\nu \epsilon_{V}\right]=\beta^{-1} R e, \\
& F_{g} \equiv \tau_{w} / \tau_{t r}=\left[N T_{L}\right]^{-1}=\beta F r \text {, } \\
& \mathcal{R}_{I} \equiv R_{g} F_{g}^{2}=\epsilon_{V} /\left[\nu N^{2}\right]=\beta \mathcal{R}_{B},
\end{aligned}
$$

with $\mathcal{R}_{B} \equiv \operatorname{ReFr}^{2}$ already defined in $\$ 2$ The difference between the two formulations in terms of $[R e, F r]$ and $\left[R_{g}, F_{g}\right]$ is the appearance of the measured efficiency of energy dissipation in turbulent flows interacting with waves in the latter case as opposed to a purely dimensional expression in the former case. $F_{g}$ and $R_{g}$ correspond to the choice of definition of Froude and Reynolds numbers in Maffioli et al. (2016) in terms of $\epsilon_{V}$ (with $\left.\epsilon_{V} /\left[N u_{r m s}^{2}\right]=F_{g}\right)$. Note that extending this second set of parameters to the rotating case, one will define $R o_{g}$ as $\left[f \tau_{t r}\right]^{-1}$ and thus $N / f=R o / F r=R o_{g} / F_{g}$ will remain the same in both formulations. It is not clear whether considering these different parameters and characteristic scales allows for a better assessment of these flows. For example, when taking this second set of parameters, $\tau_{t r}$ varies by a factor 20 within the confine of the data base in Table 1, whereas, as noted in $93 u_{r m s}$ and $L_{i n t}$, and thus $\tau_{N L}$, vary by of the order of a factor of 2 .

To illustrate this point, we examine in Fig. 9 the variation with $\left[N T_{L}\right]^{-2}=F_{g}^{+2}$ of 

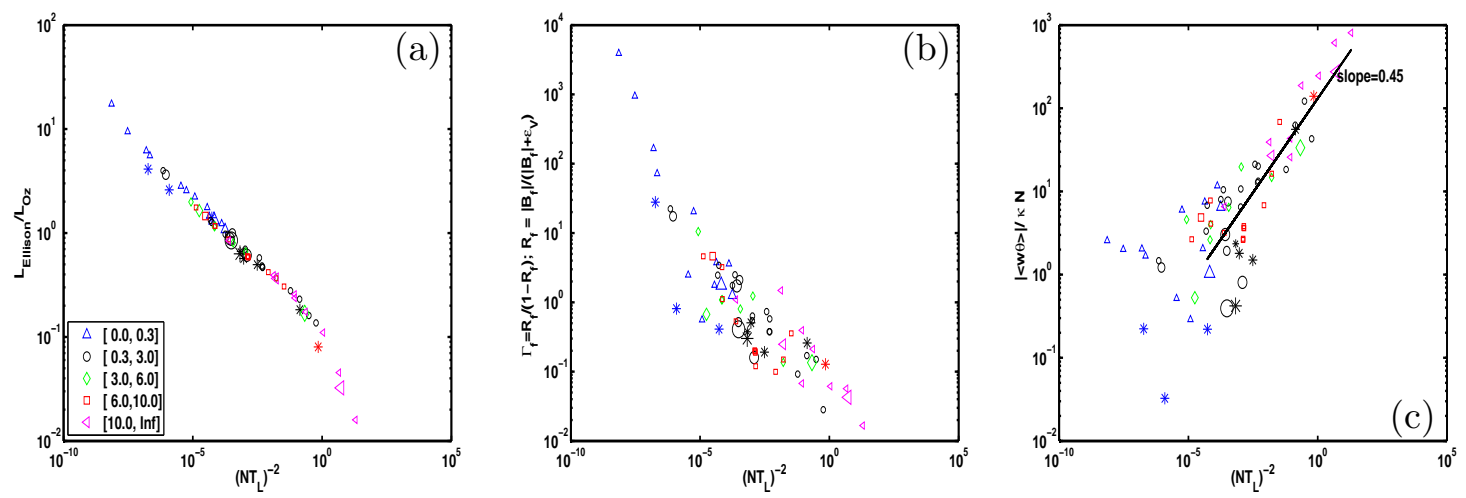

Figure 9. (Color online) Variation with $\left[N T_{L}\right]^{-2}=F_{g}^{2}$ of $L_{E l l} / L_{O z}(a)$ and of $\Gamma_{f}(b)$, as well as of the normalized eddy diffusivity $\kappa_{\rho} / \kappa(c)$, all with binning in Ro (symbols as in Fig. 1).

$L_{E l l} / \ell_{O z}(\mathrm{a})$, of $\Gamma_{f}(\mathrm{~b})$, and of the normalized diffusivity $\kappa_{\rho} / \kappa(\mathrm{c})$, with $\kappa_{\rho}=\langle w \theta\rangle N$. The choice of power of $F_{g}$ on the abscissa is to be able to compare with variations in $\mathcal{R}_{B}, \mathcal{R}_{I}$. The least-square fit for $L_{E l l} / \ell_{O z} \approx F_{g}^{-1 / 2}$ for regimes I and II is in agreement with the data in Fig. 6, with in the intermediate regime (II), $L_{E l l} / \ell_{O z} \approx F r^{-1}$, and $F_{g} \sim F r^{2}$. However, it extends now through two regimes, showing that the $F_{g}$ parameter allows to cross smoothly through these regimes, with only saturation when $F_{g} \gtrsim 1, F r \gtrsim 1$, now moving into the third regime of stratified but strong turbulence.

The two scalings that can be identified for the mixing efficiency $\Gamma_{f}$ are in agreement with previous figures, in particular with $\Gamma_{f} \sim F_{g}^{-2}$ at small $N T_{L}$, and $\Gamma_{f} \sim F_{g}^{-1}$ at large $N T_{L}$, with a cross-over at $F_{g}^{2} \approx 10^{-6}$.

Finally, the normalized eddy diffusivity again shows scaling behavior for $F_{g}$ sufficiently large. At lower $F_{g}$, it saturates to values close to unity except for three data points which are both at low Froude and low Rossby numbers, with Richardson numbers between 5 and 10 and buoyancy Reynolds numbers close to 5: these flows are in a transitional regime sensitive to fluctuations close to the threshold of instabilities.

\subsection{Appendix C: Derived dimensionless parameters}

One can also define micro-Froude and micro-Rossby numbers, $F_{\omega}$ and $R_{\omega}$, based on the effective kinetic energy dissipation rate $\epsilon_{V}$, with $\omega_{r m s}$ the $r m s$ vorticity, $\omega=\nabla \times \mathbf{u}$ :

$$
F_{\omega} \equiv\left[\frac{\epsilon_{V}}{\nu N^{2}}\right]^{1 / 2}=\frac{\omega_{r m s}}{N}, R_{\omega} \equiv\left[\frac{\epsilon_{V}}{\nu f^{2}}\right]^{1 / 2}=\frac{\omega_{r m s}}{f} .
$$

The runs of Table 1 have $11.5 \leqslant R_{\omega} \leqslant 3244$ : the intrinsic vorticity of the flow dominates the imposed rotation at small scales for all runs. Note that

$$
\mathcal{R}_{I} \equiv F_{\omega}^{2}=\epsilon_{V} /\left[\nu N^{2}\right]
$$

is called any of: the buoyancy Reynolds number (Ivey et al. 2008), or the activity parameter (Stretch et al. 2010), or the turbulence intensity parameter (de Lavergne et al. 2016). The buoyancy Reynolds number $\mathcal{R}_{B}$ is what $\mathcal{R}_{I}$ would be under the assumption that the turbulence has reached its full potential, and that the dissipation rate is equal to its dimensional expression, $\epsilon_{D}$. Thus, $\mathcal{R}_{I}$ is an expression that is compatible with a small-scale Kolmogorov isotropic energy spectrum, with $\mathcal{R}_{B}$ and $\mathcal{R}_{I}$ differing by a factor $\beta$, namely $\mathcal{R}_{B} \equiv \operatorname{ReFr}^{2}=\epsilon_{\mathbf{D}} /\left[\nu N^{2}\right]=\beta^{-1} \mathcal{R}_{I}$. In terms of ratio of characteristic length 
scales, assuming a Kolmogorov spectrum, $E_{V}(k) \sim \epsilon_{V}^{2 / 3} k^{-5 / 3}$, one can also write:

$$
\mathcal{R}_{I}=\left[\ell_{O z} / \eta\right]^{4 / 3}, \quad R_{\omega}=\left[\ell_{Z e} / \eta\right]^{2 / 3}=\beta^{1 / 2} R e^{1 / 2} R o,
$$

with as usual the dissipation and Ozmidov scales defined as $\eta=2 \pi\left[\epsilon_{V} / \nu^{3}\right]^{-1 / 4}, \ell_{O z}=$ $2 \pi \sqrt{\epsilon_{V} / N^{3}}$ (see \$2), and equivalently for rotation the Zeman scale $\ell_{Z e}=2 \pi \sqrt{\epsilon_{V} / f^{3}}$. It is thus clear that $\mathcal{R}_{I}$ represents a dimensional estimate of the ratio of inertial to dissipative forces for stratified turbulence.

\section{REFERENCES}

Barry, M., Ivey, G., Winters, K. \& Imberger, J. 2001 Measurements of diapycnal diffusivities in stratified fluids. J. Fluid Mech. 442, 267-291.

Bartello, P. 1995 Geostrophic adjustment and inverse cascade in rotating stratified turbulence. J. Atmos. Sci. 52, 4410-4428.

Billant, P. \& Chomaz, J. M. 2001 Self-similarity of strongly stratified inviscid flows. Phys. Fluids 13, 1645-1651.

Bluteau, C. E., Jones, N. L. \& Ivey, G. N. 2013 Turbulent mixing efficiency at an energetic ocean site. J. Geophys. Res. 118, 1-11.

van Bokhoven, L.J.A., Clercx, H.J.H., van Heijst, G.J.F. \& Trieling, R.R. 2009 Experiments on rapidly rotating turbulent flows. Phys. Fluids 21, 096601.

Bouffard, D. \& Boegman, L. 2013 A diapycnal diffusivity model for stratified environmental flows. Dyn. Atmosph. Oc. 61-62, 14-34.

DE BRUYN Kops, S.M. 2015 Classical scaling and intermittency in strongly stratifed Boussinesq turbulence. J. Fluid Mech. 775, 436-463.

Cambon, C., Godeferd, F. S., Nicolleau, F. \& Vassilicos, J. C. 2004 Turbulent diffusion in rapidly rotating flows with and without stable stratification. J. Fluid Mech. 499, 231255.

Cambon, C. \& JaCQuin, L. 1989 Spectral approach to non-isotropic turbulence subjected to rotation. J. Fluid Mech. 202, 295-317.

D’Asaro, E., Lee, C., Rainville, L., Harcourt, R. \& Thomas, L. 2011 Enhanced turbulence and energy dissipation at ocean fronts. Science 332, 318-322.

Davidson, P. A., Staplehurst, P. J. \& Dalziel, S. B. 2006 On the evolution of eddies in a rapidly rotating system. J. Fluid Mech. 557, 135-144.

Davis, K. A. \& Monismith, S. G. 2011 The modification of bottom boundary layer turbulence and mixing by internal waves shoaling on a barrier reef. J. Phys. Oceano. 41, 2223-2241.

de Lavergne, C., Madec, G., Le Sommier, J., Nurser, A. J. G. \& Garabato, A. C. NAVEIRA 2016 The impact of a variable mixing efficiency on the abyssal overturning. J. Phys. Ocean. 46, 663-681.

Dillon, T.M. 1982 Vertical overturns: A comparison of Thorpe and Ozmidov length scales. J. Geophys. Res. 87, 9601-9613.

Dimotakis, P.E. 2005 Turbulent mixing. Ann. Rev. Fluid Mech. 37, 329-356.

Dritschel, D.G. \& McKiver, W.J. 2015 Effect of Prandtl's ratio in geophysical turbulence. J . Fluid Mech. 777, 569-590.

Ferrari, R., Mashayek, A., McDougall, T.J., Nikurashin, M. \& Campin, J.M. 2016 Turning ocean mixing upside down. J. Phys. Oceano. 46, 2239-2261.

Finnigan, J. 1999 A note on wave-turbulence interactions and the possibility of scaling the very stable planetary boundary layer. Bound. Lay. Met. 90, 529-539.

Fleury, M. \& Lueck, R.G. 1994 Direct heat flux estimates using a towed vehicle. J. Phys. Ocean. 24, 801-818.

van Haren, H., Cimatoribus, A. A., Cyr, F. \& Gostiaux, L. 2016 Insights from a 3-D temperature sensors mooring on stratified ocean turbulence. Geophys. Res. Lett. 43 (DOI:10.1002/2016GL068032), 1-7.

Herbert, C., Marino, R., Pouquet, A. \& Rosenberg, D. 2016 Waves and vortices in the inverse cascade regime of rotating stratified turbulence with or without rotation. J. Fluid Mech. 806, 165-204. 
Herring, J. R. 1980 Statistical theory of quasi-geostrophic turbulence. J. Atmos. Sci. 37, 969-977.

Ishihara, T., Gotoh, T. \& Kaneda, Y. 2009 Study of high Reynolds number isotropic turbulence by direct numerical simulation. Ann. Rev. Fluid Mech. 41, 165-180.

Ivey, G., Winters, K. \& Koseff, J. 2008 Density stratification, turbulence but how much mixing? Ann. Rev. Fluid Mech. 40, 169-184.

Iyer, K. P., Sreenivasan, K. R. \& Yeung, P. K. 2017 Reynolds number scaling of velocity increments in isotropic turbulence. Phys. Rev. E 95, 021101(R).

Karimpour, F. \& Venayagamoorthy, S.K. 2015 On turbulent mixing in stably stratified wall-bounded flows. Phys. Fluids 27, 046603.

Kimura, Y. \& Herring, J.R. 1996 Diffusion in stably stratified turbulence. J. Fluid Mech. 328, 253-269.

Klymak, J. M., Pinkel, R. \& Rainville, L. 2008 Direct breaking of the internal tide near topography: Kaena Ridge, Hawaii. J. Phys. Oceano. 38, 380-399.

Kurien, S. \& Smith, L. M. 2014 Effect of rotation and domain aspect-ratio on layer formation in strongly stratified Boussinesq flows. J. of Turb. 15, 241-271.

Laval, J.-P., McWilliams, J. C. \& Dubrulle, B. 2003 Forced stratified turbulence: Successive transitions with reynolds number. Phys. Rev. E 68, 036308.

LELONG, M-P. \& RILEY, J.J. 1991 Internal wave-vortical mode interactions in strongly stratified flows. J. Fluid Mech. 232, 1-19.

Lelong, M-P. \& Sundermeyer, M. 2005 Geostrophic adjustment of an isolated diapycnal mixing event and its implications for small-scale lateral dispersion. J. Phys. Oceano. 35, $2352-2367$.

LiNDBORG, E. 2006 The energy cascade in a strongly stratified fluid. J. Fluid Mech. 550, 207242.

Lindborg, E. \& Brethouwer, G. 2008 Vertical dispersion by stratified turbulence. J. Fluid Mech. 614, 303-314.

Liu, H.L., Yudin, V. \& Roble, R. 2013 Day-to-day ionospheric variability due to lower atmosphere perturbations. Geophys. Res. Lett. 40, 665-670.

Lozovatsky, I.D. \& Fernando, H.J.S. 2013 Mixing efficiency in natural flows. Phil. Trans. A 371, 20120213.

Luketina, D. \& Imberger, J. 1989 Turbulence and entrainment in a buoyant surface plume. J. Geophys. Res. 94, 12619-12636.

Maffioli, A., Brethouwer, G. \& Lindborg, E. 2016 Mixing efficiency in stratified turbulence. J . Fluid Mech. 794, R3.

Maffioli, A. \& Davidson, P.A. 2016 Dynamics of stratified turbulence decaying from a high buoyancy Reynolds number. J. Fluid Mech. 786, 210-233.

Marino, R., Pouquet, A. \& Rosenberg, D. 2015a Resolving the paradox of oceanic largescale balance and small-scale mixing. Phys. Rev. Lett. 114, 114504.

Marino, R., Rosenberg, D., Herbert, C. \& Pouquet, A. $2015 b$ Interplay of waves and eddies in rotating stratified turbulence and the link with kinetic-potential energy partition. EuroPhys. Lett. 112, 49001.

Mashayek, A. \& Peltier, W. R. 2013 Shear-induced mixing in geophysical flows: does the route to turbulence matter to its efficiency? J. Fluid Mech. 725, 216 - 261.

Mashayek, A., Salehipour, H., Bouffard, D., Caulfield, C.P., Ferrari, R., Nikurashin, M., Peltier, W.R. \& Smyth, W.D. 2017 Efficiency of turbulent mixing in the abyssal ocean circulation. Geophys. Res. Lett. 44, 6296-6306.

Mater, B.D., Schaad, S.M. \& Venayagamoorthy, S.K. 2013 Relevance of the Thorpe length scale in stably stratified turbulence. Phys. Fluids 25, 076604.

Mater, B.D. \& Venayagamoorthy, S.K. 2014 The quest for an unambiguous parameterization of mixing efficiency in stably stratified geophysical flows. Geophys. Res. Lett. 41, 4646-4653.

McWilliams, J. 2016 Submesoscale currents in the ocean. Proc. Roy. Soc. A 472, 2016.0117.

MÉtAis, O. \& HERRing, J. 1989 Numerical simulations of freely evolving turbulence in stably stratified fluids. J. Fluid Mech. 202, 117-148.

Mininni, P.D. \& Pouquet, A. 2017 Generation of turbulence through frontogenesis in sheared stratified flows. Submitted to Phys. Rev. F, ArXiv 1706.10287. 
Mininni, P.D., Rosenberg, D. \& Pouquet, A. 2012 Isotropization at small scale of rotating helically driven turbulence. J. Fluid Mech. 699, 263-279.

Mininni, P.D., Rosenberg, D., Reddy, R. \& Pouquet, A. 2011 A hybrid MPI-OpenMP scheme for scalable parallel pseudospectral computations for fluid turbulence. Parallel Computing 37, 316-326.

Monin, A. S. \& Yaglom, A. M. 1979 Statistical Fluid Mechanics. MIT Press, Cambridge.

Osborn, T.R. 1980 Estimates of the local rate of vertical diffusion from dissipation measurements. J. Phys. Oceano. 10, 83-89.

Paoli, R., Thouron, O., Escobar, J., Picot, J. \& Cariolle, D. 2014 High-resolution largeeddy simulations of stably stratified flows: application to subkilometer-scale turbulence in the upper troposphere-lower stratosphere. Atm. Chem. Phys. 14, 5037-5055.

Patterson, M.D., Caulfield, C.P., McElwaine, J.N. \& Dalziel, S.B. 2006 Timedependent mixing in stratified Kelvin-Helmholtz billows: Experimental observations. Geophys. Res. Lett. 33, L15608.

Peltier, W. \& Caulfield, C. 2003 Mixing efficiency in stratified shear flows. Ann. Rev. Fluid Mech. 35, 135.

PhILlips, O.M. 1972 Turbulence in a strongly stratified fluid: Is it unstable? Deep Sea Res. 19, 79-81.

Pouquet, A. \& Marino, R. 2013 Geophysical turbulence and the duality of the energy flow across scales. Phys. Rev. Lett. 111, 234501.

Pouquet, A., Marino, R., Mininni, P. D. \& Rosenberg, D. 2017 Dual constant-flux energy cascades to both large scales and small scales. Phys. Fluids 29 (111108).

Praud, O., Sommeria, J. \& Fincham, A. 2006 Decaying grid turbulence in a rotating stratified fluid. J. Fluid Mech. 547, 389-412.

Pumir, A., Xu, H. \& Siggia, E. D. 2016 Small-scale anisotropy in turbulent boundary layers. J. Fluid Mech. 804, 5-23.

Riley, J. J. \& DeBruynKops, S. M. 2003 Dynamics of turbulence strongly influenced by buoyancy. Phys. Fluids 15, 2047-2059.

Rorai, C., Mininni, P.D. \& Pouquet, A. 2014 Turbulence comes in bursts in stably stratified flows. Phys. Rev. E 89, 043002.

Rosenberg, D., Marino, R., Herbert, C. \& Pouquet, A. 2016 Variations of characteristic time-scales in rotating stratified turbulence using a large parametric numerical study. Eur. Phys. J. E 39, 8.

Rosenberg, D., Marino, R., Herbert, C. \& Pouquet, A. 2017 Correction to: Variations of characteristic time scales in rotating stratified turbulence using a large parametric numerical study. Eur. Phys. J. E 40, 87.

Rosenberg, D., Pouquet, A., Marino, R. \& Mininni, P.D. 2015 Evidence for BolgianoObukhov scaling in rotating stratified turbulence using high-resolution direct numerical simulations. Phys. Fluids 27, 055105.

Rubinstein, R., Clark, T. T. \& Kurien, S. 2017 Leith diffusion model for homogeneous anisotropic turbulence. Comp. Fluids 151, 108-114.

Salehipour, H. \& Peltier, W.R. 2015 Diapycnal diffusivity, turbulent Prandtl number and mixing efficiency in Boussinesq stratified turbulence. J. Fluid Mech. 775, 464-500.

Shin, L., Koseff, J., Ivey, G. \& Ferziger, J. 2005 Parameterization of turbulent fluxes and scales using homogeneous sheared stably stratified turbulence simulations. J. Fluid Mech. 525, 193-214.

Smyth, W.D., Moum, J.N. \& Caldwell, D.R. 2001 The efficiency of mixing in turbulent patches: Inferences from direct simulations and microstructure observations. J. Phys. Oceano. 31, 1969-1992.

Sozza, A., Boffetta, G., Muratore-Ginanneschi, P. \& Musacchio, S. 2015 Dimensional transition of energy cascades in stably stratified forced thin fluid layers. Phys. Fluids 27, 035112 .

Stacey, M., Monismith, S. \& Burau, J. 1999 Observations of turbulence in a partially stratified estuary. J. Phys. Oceano. 29, 1950-1970.

Staquet, C. \& Sommeria, J. 2002 Internal gravity waves: From instabilities to turbulence. Ann. Rev. Fluid Mech. 34, 559. 
Stillinger, D., Helland, K. \& van Atta, C. 1983 Experiments on the transition of homogeneous turbulence to internal waves in a stratified fluid. J. Fluid Mech. 131, 91-122.

Stretch, D.D., Rottman, J., Venayagamoorthy, S.K., Nomura, K. \& Rehmann, C. R. 2010 Mixing efficiency in decaying stably stratified turbulence. Dyn. Atm. Oc. 49, 25-36.

Sukoriansky, S., Galperin, B. \& Staroselsky, I. 2005 A quasinormal scale elimination model of turbulent flows with stable stratification. Phys. Fluids 17, 085107.

Thorpe, S.A. 1987 Transitional phenomena and the development of turbulence in stratified fluids: a review. J. Geophys. Res. 92, 5231-5248.

Venayagamoorthy, S.K. \& Koseff, J.R. 2016 On the flux Richardson number in stably stratified turbulence. J. Fluid Mech. 798, R1-R10.

Waite, M. \& Bartello, P. 2006 The transition from geostrophic to stratified turbulence. J. Fluid Mech. 568, 89-108.

Wells, M., Cenedese, C. \& Caulfield, C.P. 2010 The relationship between flux coefficient and entrainment ratio in density currents. J. Phys. Oceano. 40, 2713-2727.

Zakharov, V. E., L'vov, V. S. \& Falkovich, G. 1992 Kolmogorov spectra of turbulence: Wave turbulence. Springer, Non-linear dynamics .

Zilitinkevich, S.S., Elperin, T., Kleeorin, N., Rogachevskit, I. \& Esau, I. 2013 A hierarchy of energy- and flux-budget (EFB) turbulence closure models for stably-stratified geophysical flows. Bound.-Layer Meteorol. 146, 341-373.

Zilitinkevich, S. S., Elperin, T., Kleeorin, N., Rogachevskit, I., Esau, I., Mauritsen, T. \& Miles, M. W. 2008 Turbulence energetics in stably stratified geophysical flows: Strong and weak mixing regimes. Quart. J. Met. Roy. Soc. 134, 793-799. 